\title{
Comparison of Filtration Performance Between Neat and Plasma-Treated PAN / MgO Nanofibers in the Removal of 10 To 1000 Nm Particles
}

Farideh Golbabaei, Professor, Department of Occupational Health, School of Public Health, Tehran University of Medical Sciences, Tehran, Iran.

Majid Habibi Mohraz, Assistant Professor, Department of Occupational Health, School of Public Health, Hamedan University of Medical Sciences, Hamedan, Iran.

Rasoul Yarahmadi, Associate Professor, Department of Occupational Health, School of Public Health, Iran University of Medical Sciences, Tehran, Iran.

Asghar Sadighzadeh, Nuclear Fusion Research Center, Atomic Energy Organization, Tehran, Iran.

Hamzeh Mohammadi, PhD Student, Department of Occupational Health, School of Public Health, Tehran University of Medical Sciences, Tehran, Iran.

(1) Somayeh Farhang Dehghan, $\left({ }^{\star}\right.$ Corresponding author), Assistant Professor, Environmental and Occupational Hazards Control Research Center, School of Public Health and Safety, Shahid Beheshti University of Medical Sciences, Tehran, Iran. somayeh.farhang@gmail.com

\section{Abstract}

Background and aims: The present study aims to compare the filtration performance of the neat hybrid electrospun nanofibers, consisting of polyacrylonitrile (PAN) polymer and magnesium oxide $(\mathrm{MgO})$ nanoparticles and the plasma treated ones in removing fine particles from the air stream.

Methods: The upper surface of the nanofibers, were processed by cold-plasma with a radio frequency power supply (13.56 MHz with a power of 20 watts), argon gas and operating pressure of 0.2 torr. Initial efficiency tests for numerical removal of particles have been done in accordance with standards ISO 29463 and EN 779. Pressure drop and quality factors were determined for the fabricated media. In order to confirm the presence of magnesium oxide nanoparticles in the nanofibers, X-Ray Diffraction pattern (XRD) was prepared. Analysis algorithms of SEM images, were used to calculate the porosity of filters, using the MATLAB software.

Results: The mean initial efficiency of the neat and plasma-treated media was, $90.77 \pm 6.7 \%$ and $73.66 \pm 8.86 \%$ for collecting particles from 10 to $1000 \mathrm{~nm}$, respectively. The initial mean pressure drop of the neat and treated media at the test face velocity was, $78.22 \pm 3.11$ pa and $22.00 \pm 2.33$ pa, respectively, and their mean quality factor for collecting 10 to $1000 \mathrm{~nm}$ particles was, 0.029 and 0.010 (Pascal/1), respectively.

Conclusion: By performing plasma treatment, collection efficiency of particles decreased, but with a significant decrease in pressure drop, the neat media ultimately presented a higher score of quality factor in comparison with the treated ones.

Conflicts of interest: None

Funding: Tehran University of Medical Sciences

\section{Keywords \\ Filtration \\ Nanofiber \\ Plasma-treated \\ Performance \\ Nanoparticle}

Received: 2019/07/19

Accepted : 2020/05/3 


\section{INTRODUCTION}

Polymer nanofibers are increasingly used in various fields, due to their large surface area per unit of mass and their small sizes, which are often produced by electrospinning techniques. Mixing the polymer nanofibers with the metal oxide nanoparticles such as, $\mathrm{MgO}$ and other oxides, has been considered by some researchers for air purification applications. This is due to the unique ability of these nanoparticles to clean a wide variety of toxic gases, such as chemical pollutants, biological pollutants (viruses, bacteria), pesticides, and many more [1]. Hybrid nanofibers (for example, a combination of polymers and nanoparticles) can present multiple functions in the simultaneous purification of aerosols, bioaersols, and gaseous pollutants. The electrospun nanofiber mat itself, is soft and brittle, on the other hand, due to the high pressure drop across its surface, nanofiber mat is not commonly used as an air filter media [2]. In an effort to provide good structural support, without altering the nanofiber properties and preventing this pressure drop, the nanofiber composites have been introduced. This means that, nanofibers are coated on a rigid substrate to form a composite. However, one of the major challenges in the composite structure, is the good adhesion of the nanofibers to the substrate and the satisfactory durability of the nanofibers against bending and abrasion. Among the various methods available for the surface treatment, as a surface modification technique, Plasma treatment has introduced itself as a clean and environmentally friendly product, to overcome these problems [3]. In addition, different types of surface modifications can be easily designed by selecting the gas for the plasma production. Surface activation is achieved through plasma treatment, by replacing or adding chemical groups and so altering the surface's properties. Studies have shown, that by plasma, the polymer's surface can be made much more efficient and effective. Plasma polymers are composed of various active species, including free radicals and ions. The process is done without generating any environmental pollution [4]. The present study, aims to compare the filtration performance of the neat hybrid nanofibers consisting of polyacrylonitrile (PAN) polymer, the magnesium oxide $(\mathrm{MgO})$ nanoparticles and plasma treated ones, in removing fine particles from the air stream.

\section{METHODOLOGY}

In order to fabricate the filter media containing the nanofiber layer, the nozzle-based solution electrospinning technique was applied. Materials used to prepare the electrospinning solution included: the polyacrylonitrile polymer (PAN), solvent $\mathrm{N}$, $\mathrm{N}$-dimethyl formamide (DMF) and magnesium oxide nanoparticles $(\mathrm{MgO})$. They were purchased from Polyacryle Co. (Isfahan, Iran), Merck Co. (Darmstadt, Germany) and Merck Co., respectively. The concentration of PAN / MgO solution was 16\% wt. in 3: 1 ratio in DMF solvent. Nanofibers were fabricated with nozzle solution electrospinning machine (Nano-Meghyas R\&D Co, Iran) in the following conditions: Applied voltage: $20 \mathrm{kV}$, needle to drum collector distance: $10 \mathrm{~cm}$, ambient temperature: 20-25 ${ }^{\circ} \mathrm{C}$, electrospinning duration: 30 minutes, flow rate: $1 \mathrm{ml} / \mathrm{h}$; Needle type: 18 gauge and the rotation speed of the drum: 500-1000 rpm. All electrospinning variables were selected based on the optimal

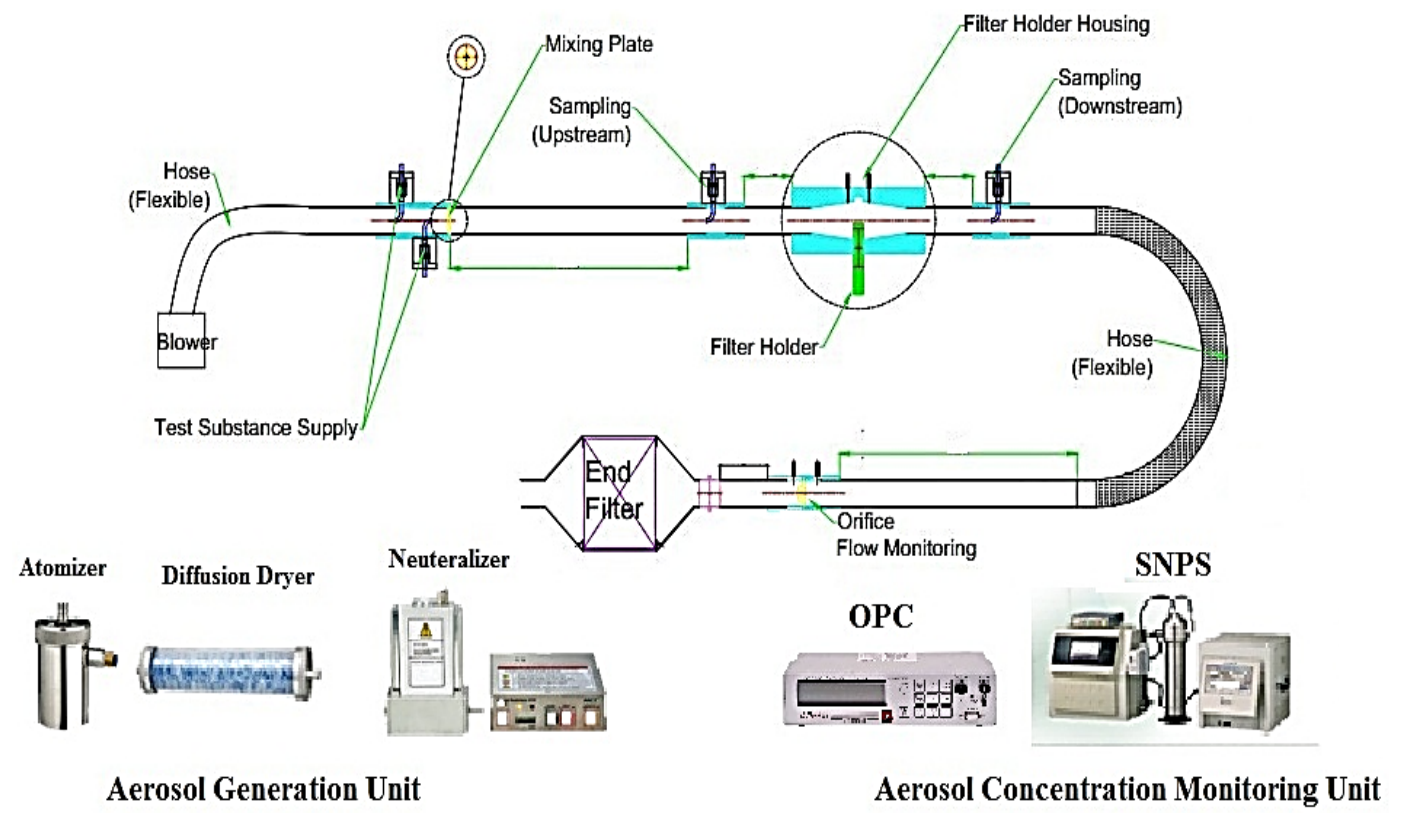

Fig. 1. Schematic diagram of the filter test rig [8] 
models in the author's previous studies. During the electrospinning process, the nanofibers were deposited on a polypropylene spun bond substrate, with a basis weight of $17 \mathrm{~g} / \mathrm{m}^{2}$, a filament diameter of $3.5-11.5 \mu \mathrm{m}$ and a thickness of $100 \mu \mathrm{m}$.

For the plasma treatment, the substrate coated with PAN / MgO nanofiber layer was used. The samples were placed on the central plate, as a grounded negative electrode of a cold plasma device (manufactured by the Atomic Energy Organization of Iran) which acted as a cathode. The cathode electrode $(15 \mathrm{~cm}$ in diameter) was located $5 \mathrm{~cm}$ away from the electrical-discharge electrode $(10 \mathrm{~cm}$ in diameter as the anode), which was connected to the radio frequency (RF) generator. The surface of the samples were treated for 10 minutes, under cold plasma with the RF power supply (13.56 MHz with a power of 20 watts) with the argon gas and an operating pressure of 0.2 Tor.

Initial pressure drop and removal efficiency of particles less than $1000 \mathrm{~nm}$, was measured according to the ISO 29463: 2011 standards [5] in the test rig (Fig. 1), which was designed based on ASHREA52.2: 2007[6]. The filter media was placed in a holder (open cross-sectional area of $28.26 \mathrm{~cm}^{2}$ ) and then, the required air flow was set based on the test face velocity $(10 \mathrm{~cm} / \mathrm{s})$. The filtration efficiency test was performed by injecting KCL particles, in the size ranges of $10 \mathrm{~nm}$ to $1000 \mathrm{~nm}$ in the test rig, by the HCT-Atomizer 4810-South Korea, and counting the filter media's particle's downstream and upstream by the Nanoparticle Scanning Spectrometer (SNPS) (HCT -SNPS-20N- South Korea ) and Optical Particle Counter (OPC) (Grimm -1.109- Germany). Particles were emitted from the atomizer of the diffusion dryer (HCT-4920-South Korea) and neutralizer (HCTXRC03 -South Korea) to remove moisture from the particles and neutralize their electrical charges, respectively. In the test rig, High-Efficiency Particulate Air filters (HEPA) were used to purify the inlet air and dilute the particle's concentration. Initial pressure drop was measured by the differential digital manometer and quality factors were determined for the produced media. Temperature and relative humidity of the test airflow were recorded. Seven samples were taken from the upstream and six samples from the downstream media (sampling time was two minutes and fifteen seconds at a time) and the results were recorded in the SNPS and OPC software systems. The results obtained from the counting the upstream and downstream were averaged and then the collection efficiency was calculated. It should be noted that at least 4 samples of each type of the media were tested for assessing the filtration performance. The calculation of Coefficient of the Variation (CV) and uncertainty of the results, was performed according to EN 779: 2012 standard
[7]. All tests were performed in the temperature ranges of 20 to $25{ }^{\circ} \mathrm{C}$, relative humidity of 25 to $50 \%$ and atmospheric pressure of about 1005 mbar. All the calculations and the graph's drawings were done with the help of Microsoft Excel 2010 software.

Using the Scanning Electron Microscopy (SEM) (Philips-XL 30-USA), the fiber morphology studies and fiber diameter determinations were performed. Elemental detection of the magnesium oxide nanoparticles in $\mathrm{PAN} / \mathrm{MgO}$ nanofiber media was performed by Wavelength- dispersive X-ray spectroscopy (WDX). The X-Ray Diffraction (XRD) (STOE-STADIP 'Germany) was also used, to confirm the presence of magnesium oxide particles in the nanofibers. SEM image analysis algorithms were done through MathWorks software, Version 7 (MATLAB) to calculate the percentage of the media's porosity. Measurements of the media thickness was done by using a digital caliper (ASIMETO- 307-56-3 6" ABS- Hong Kong).

\section{RESULT}

Table (1) reveales the results for the mean initial efficiency, pressure drop and quality factors of the filter media, for 80 to $250 \mathrm{~nm}$ particles (according to the standard ISO 29463-3), as well as the mean overall efficiency for the particles below 1 micron, most penetrating particle size (MPPS) of the media and their quality factors for 100 and $300 \mathrm{~nm}$ particles. On average, the highest filtration efficiencies and the lowest pressure drops were obtained from neat and treated PAN/MgO media, respectively. However, the highest quality factors belongs to treated media; the efficiency of the particle collection was reduced by the plasma treatment. Indeed, with a significant reduction in the pressure drop of the treated media, it's quality factors eventually became more significant than that of neat media. The MPPS was $100 \mathrm{~nm}$ for both types of the media. It should be noted that, the $\mathrm{CV}$ and uncertainty results of less than $5-10 \%$ is considered as an acceptable and reiable result.

Dimensional characteristics of the studied fibers in neat and plasma-treated media, along with their size distribution and the corresponding SEM images are given in Table (2). In general, neat PAN / MgO nanofibers had smaller fiber diameters and the plasma treatment could lead to the increase in the fiber's diameter. The mean diameter of the substrate fibers were $22.37 \pm 3.66$ microns

Structural characteristics of the neat and plasmatreated media, including their morphology, thickness, basis weight, and porosity are listed in Table (3). In cases where the ratio of the standard deviation of the fiber diameter to the mean diameter is less than 0.3 , the morphological type is considered as a uniform and otherwise, a non-uniform. The studied media showed 
Golbabaei F, et al.

Table 1. Results of filtration performance of the studied media in removal of particles below 1 micron.

\begin{tabular}{|c|c|c|c|}
\hline \multirow{2}{*}{$\begin{array}{l}\text { Particle size } \\
\text { (nm) }\end{array}$} & \multirow{2}{*}{ Parameter } & \multicolumn{2}{|c|}{ Media } \\
\hline & & Neat & Treated \\
\hline \multirow{3}{*}{80} & Mean $( \pm$ SD) of initial efficiency (\%) & $82.75 \pm 2.64$ & $53.43 \pm 2.12$ \\
\hline & Uncertainty (\%) & 2.76 & 2.21 \\
\hline & Coefficient of variation (\%) & 3.19 & 3.96 \\
\hline \multirow{4}{*}{100} & Mean ( \pm SD) of initial efficiency (\%) & $81.60 \pm 2.61$ & $51.57 \pm 1.76$ \\
\hline & Uncertainty (\%) & 2.73 & 1.84 \\
\hline & Coefficient of variation (\%) & 3.19 & 3.41 \\
\hline & Mean ( \pm SD) of initial efficiency (\%) & $83.07 \pm 2.35$ & $53.51 \pm 1.52$ \\
\hline \multirow[t]{3}{*}{125} & Uncertainty (\%) & 2.46 & 1.59 \\
\hline & Coefficient of variation (\%) & 2.82 & 2.84 \\
\hline & Mean ( \pm SD) of initial efficiency (\%) & $83.54 \pm 2.11$ & $56.45 \pm 1.87$ \\
\hline \multirow[t]{3}{*}{160} & Uncertainty (\%) & 2.21 & 1.96 \\
\hline & Coefficient of variation (\%) & 2.52 & 3.31 \\
\hline & Mean ( \pm SD) of initial efficiency $(\%)$ & $86.87 \pm 1.47$ & $63.30 \pm 1.16$ \\
\hline \multirow[t]{3}{*}{200} & Uncertainty (\%) & 1.82 & 1.21 \\
\hline & Coefficient of variation (\%) & 2.00 & 1.83 \\
\hline & Mean $( \pm$ SD) of initial efficiency (\%) & $88.36 \pm 1.01$ & $67.37 \pm 0.58$ \\
\hline \multirow[t]{2}{*}{250} & Uncertainty (\%) & 1.05 & 0.84 \\
\hline & Coefficient of variation (\%) & 1.14 & 0.86 \\
\hline \multicolumn{2}{|c|}{ Mean initial efficiency for 80 to $250 \mathrm{~nm}$ particles (\%) } & $88.36 \pm 2.28$ & $57.61 \pm 6.19$ \\
\hline \multicolumn{2}{|c|}{ Mean initial penetration for 80 to $250 \mathrm{~nm}$ particles (\%) } & 15.64 & 42.39 \\
\hline \multicolumn{2}{|c|}{ Mean initial pressure drop $(\mathrm{Pa})$} & $78.22 \pm 3.11$ & $22.00 \pm 2.23$ \\
\hline \multicolumn{2}{|c|}{ Mean quality factor for 80 to $250 \mathrm{~nm}$ particles ( $\mathrm{Pa}-1)$} & 0.0237 & 0.0390 \\
\hline \multicolumn{2}{|c|}{ Mean initial efficiency for particles $300 \mathrm{~nm}$} & $90.80 \pm 1.26$ & $72.15 \pm 2.16$ \\
\hline \multicolumn{2}{|c|}{ Mean quality factor $(\mathrm{Pa}-1)$ for particles $300 \mathrm{~nm}$} & 0.0637 & 0.0305 \\
\hline \multicolumn{2}{|c|}{ Mean quality factor $(\mathrm{Pa}-1)$ for particles $100 \mathrm{~nm}$} & 0.0329 & 0.0216 \\
\hline \multicolumn{2}{|c|}{ Mean initial efficiency for particles below $1000 \mathrm{~nm}$} & $90.37 \pm 6.07$ & $73.60 \pm 8.86$ \\
\hline \multicolumn{2}{|c|}{ Most penetrating particle size $(\mathrm{nm})$} & 101.8 & 101.8 \\
\hline
\end{tabular}

Table 2. Dimensional characteristics of the neat PAN / MgO nanofibers and the plasma treated ones.

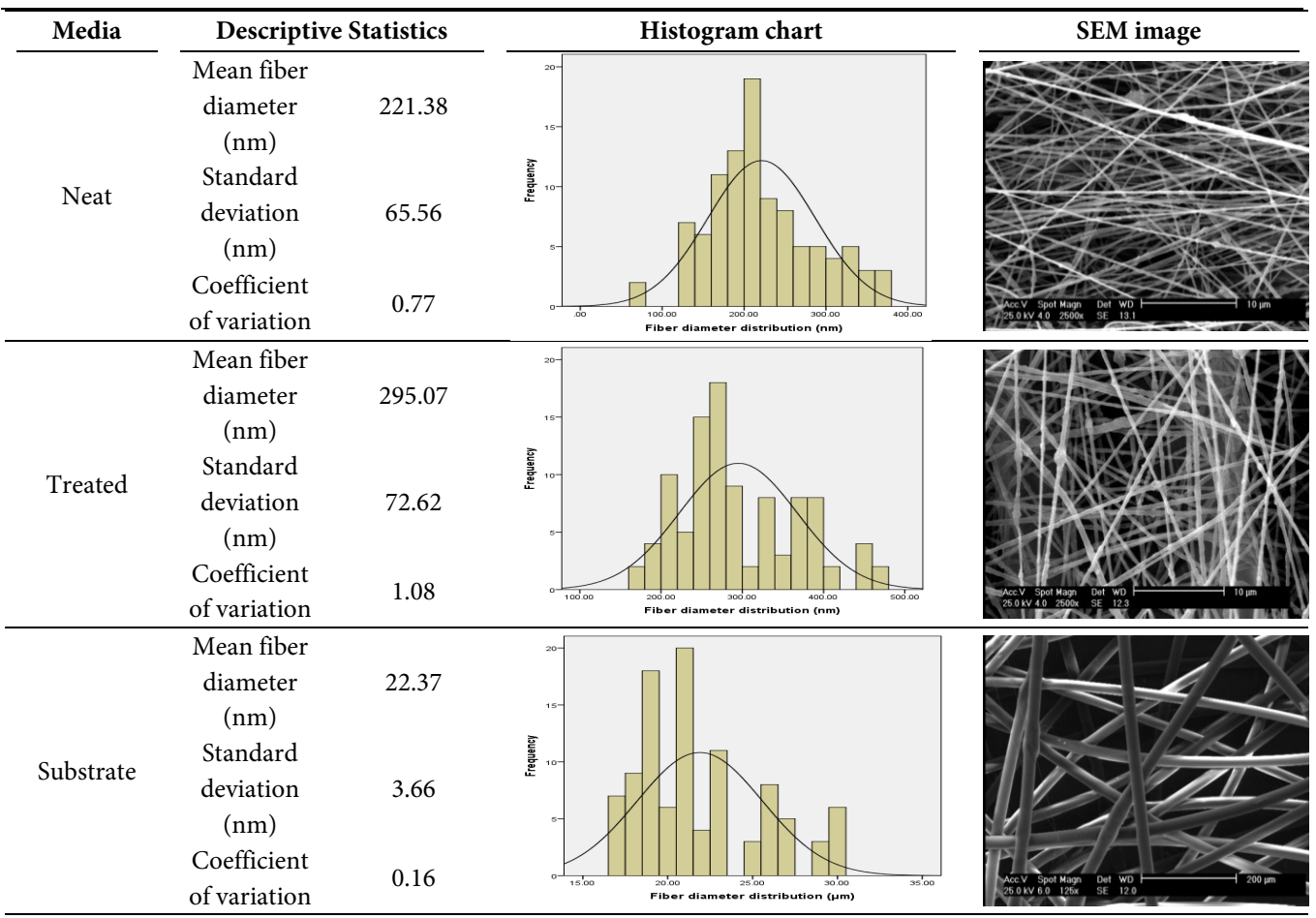


Comparison of Filtration Performance Between Neat ...

Table 3. Structural characteristics of the neat PAN / MgO nanofibers and plasma treated ones.

\begin{tabular}{ccccc}
\hline \multirow{2}{*}{ Media } & Morphology & $\begin{array}{c}\text { Thickness of media } \\
(\mathbf{m m})\end{array}$ & $\begin{array}{c}\text { Basis weight of media } \\
(\mathbf{g s m})\end{array}$ & Porosity (\%) \\
\hline Neat & Beaded $^{*}$ & 0.115 & 17.58 & 41 \\
Treated & Beaded & 0.115 & 17.58 & 48 \\
\hline
\end{tabular}

${ }^{\star}$ Morphology of beaded fibers is obtained directly from SEM images.

slight differences, in the thickness and basis weight, while they had different conditions for porosity.

According to the XRD pattern, three reflection peaks corresponding to the pure $\mathrm{MgO}$ crystals appeared at $2 \theta=36.8^{\circ}, 2 \theta=42.8^{\circ}$ and $2 \theta=62.2^{\circ}$, suggesting that the nanofibers contain pure $\mathrm{MgO}$.

\section{CONCLUSION}

In terms of morphology, the neat PAN / MgO nanofibers had lower porosity and a higher packing density compared to the plasma-treated ones, which increased the air flow resistance of the media, reduced it's air permeability, and increased the pressure drop. Increased pressure drop of the neat PAN / $\mathrm{MgO}$ nanofibers compared to the treated ones, can also be due to the smaller diameter of their fibers. The plasma treatment, reduced the particle collection efficiency of the media. However, the quality factors of the treated media were generally higher than the neat one. The higher collection efficiency of the neat media can be attributed to their smaller diameter, higher packing density, and their beaded morphology. According to the classic filtration theory, the efficiency is directly related to the thickness and packing density of the media, and is inversely related to the fiber diameter and the porosity. Fibers with the smaller diameters have higher surface areas, higher packing densities and larger pore sizes, which results in an increased filtration capacity of the media. On the other hand, the fibers which are larger in diameter, are usually bulkier, more porous, and have a higher air permeability and a lower pressure drop.
Conventional microfiber media, have a minimum particle collection efficiency of 100 to 500 nanometers, called the Most Penetrating Particle Size (MPPS). Their efficiency can be increased by the use of nanofibers. For different types of media, the results of the MPPS showed that, the mean for the two different types of the media is the same; $101.8 \mathrm{~nm}$.Various studies indicate that, lowering the fiber diameter and increasing the basis weight, causes the MPPS to move to a smaller size range, which is probably due to the obtained results. The mean difference in the fiber diameter, in both the neat and treated media, was not significant enough to cause a change in MPPS. In conclusion, the highest percentage of the filtration efficiency and the lowest pressure drop, were obtained from the neat and plasma-treated PAN / MgO media, respectively. However, the treated media had the highest quality factor. By performing plasma treatment, collection efficiency of the particles decreased, but with a significant decrease in the pressure drop, the treated media ultimately presented a higher score in quality factor than the neat one. Therefore, the plasma treatment can be a good option for surface modification of the filter media, although the definitive conclusion depends on additional tests such as, mechanical resistance of the media, adhesion of the layer and so on.

\section{CONFLICT OF INTEREST}

The authors declare that there are no conflicts of interest regarding the publication of this manuscript.

How to cite this article:

Farideh Golbabaei, Majid Habibi Mohraz, Rasoul Yarahmadi, Asghar Sadighzadeh, Hamzeh Mohammadi, Somayeh Farhang Dehghan. Comparison of Filtration Performance Between Neat and Plasma-Treated PAN / MgO Nanofibers in the Removal of 10 To 1000 Nm Particles. Iran Occupational Health. 2021 (01 Feb);18:4. 


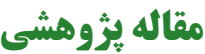

http://ioh.iums.ac.ir

\section{مقايسه عملكرد فيلتراسيون نانوالياف PAN/MgO ساده و يرداز ش شده با پإسما در حذف ذرات

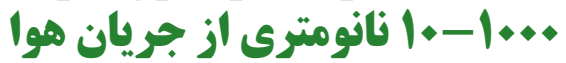

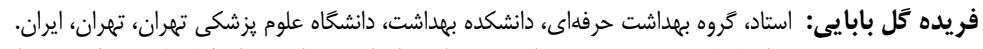

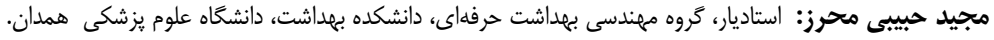

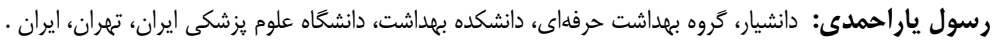

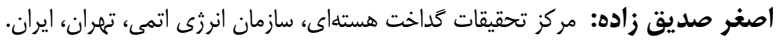

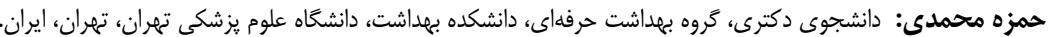

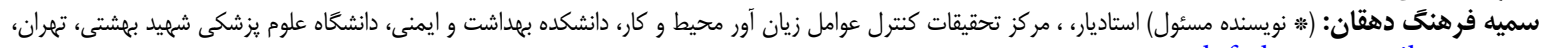
ايران . somayeh.farhang@gmail.com

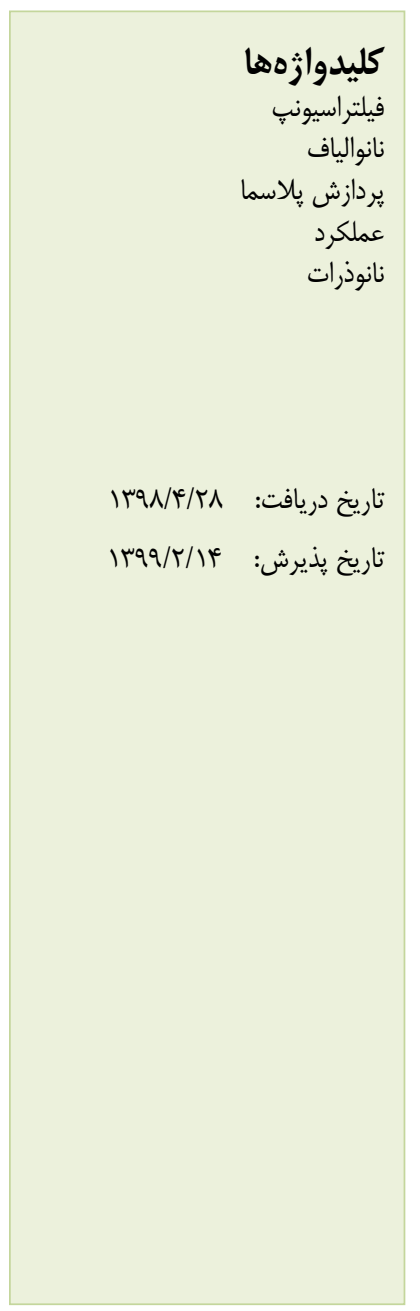

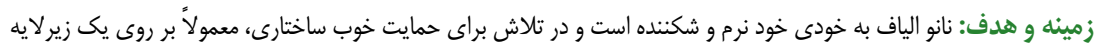

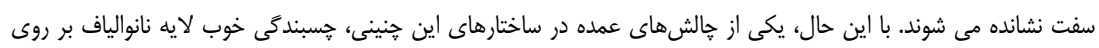

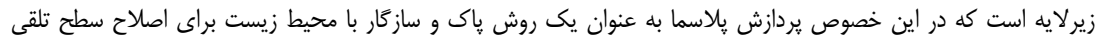

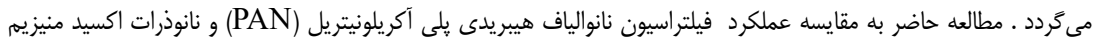

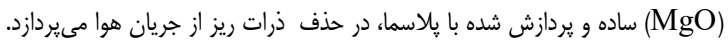

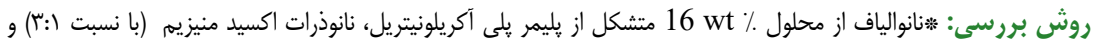

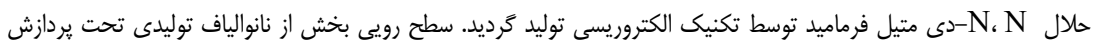

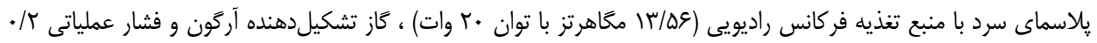

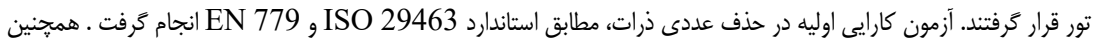

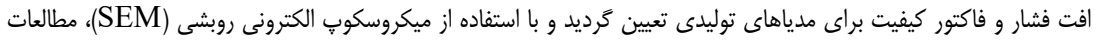

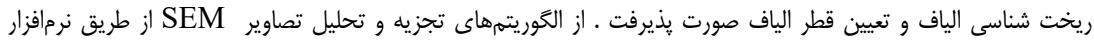

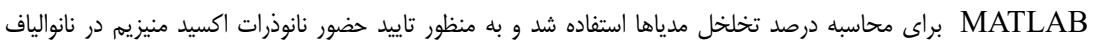
PAN/MgO

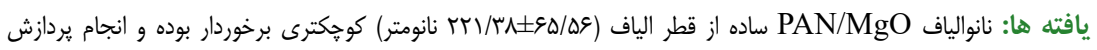

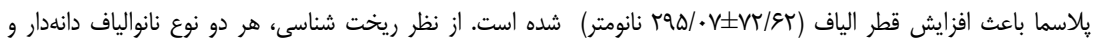

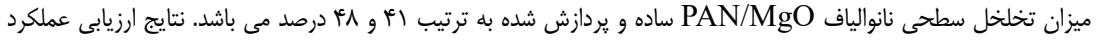

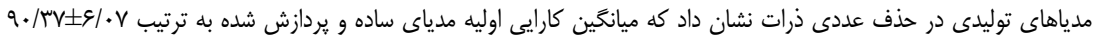

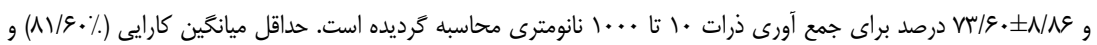

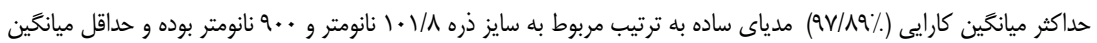

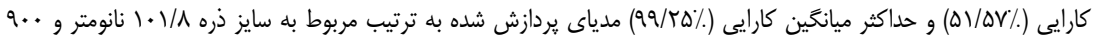

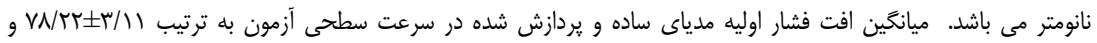

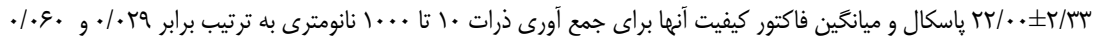

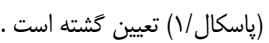

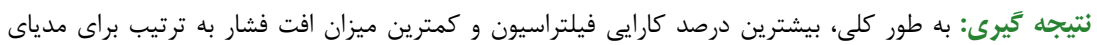

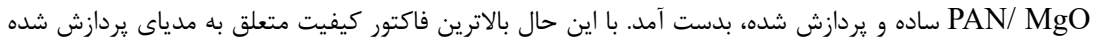

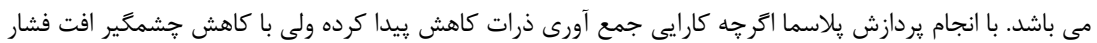

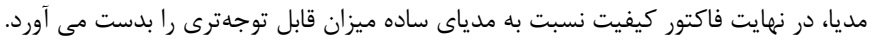

$$
\text { تعارض منافع: كزارش نشده /ست. }
$$

منبع حما يت كنتده: دانشكاه علوم بزنشكى تهران.

شيوه استناد به اين مقاله:

Farideh Golbabaei, Majid Habibi Mohraz, Rasoul Yarahmadi, Asghar Sadighzadeh, Hamzeh Mohammadi, Somayeh Farhang Dehghan. Comparison of Filtration Performance Between Neat and Plasma-Treated PAN / MgO Nanofibers in the Removal of 10 To 1000 Nm Particles. Iran Occupational Health. 2021 (01 Feb);18:4. 
نانوليفى نشانده شده (كاميوزيت) معرفى شدهاند. يعنى

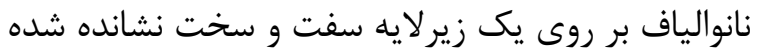

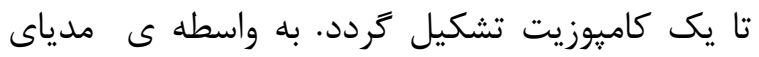

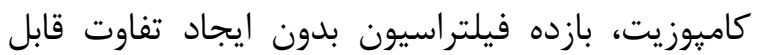

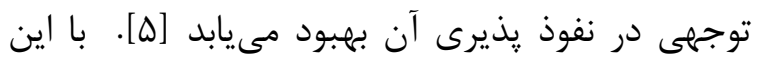

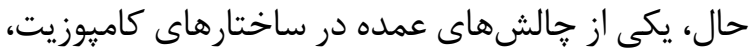

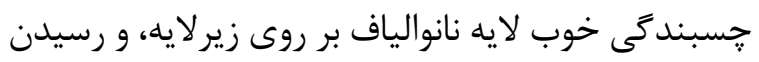

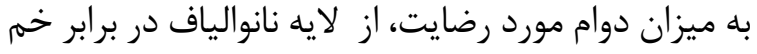

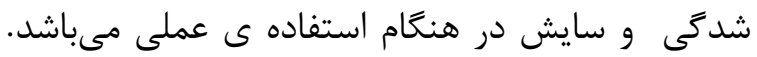

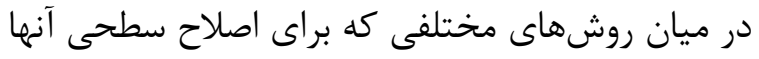

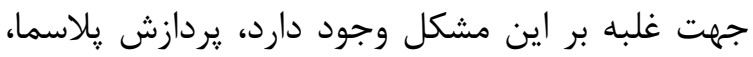

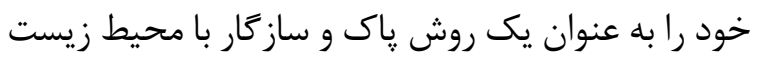

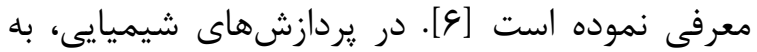
عنوان روشى ماى مرسوم و سنتى اصلاح سطحى، مقدي مقدار

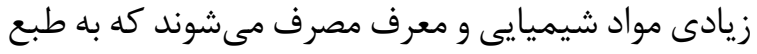

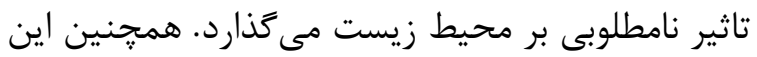

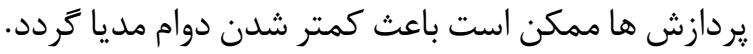

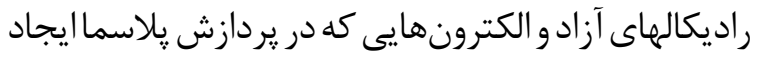

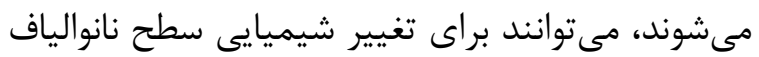

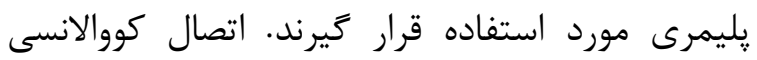

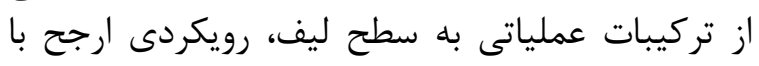

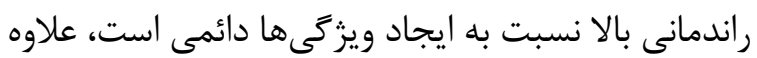

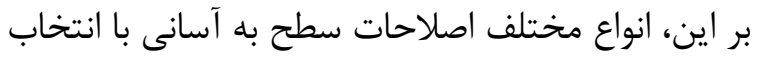

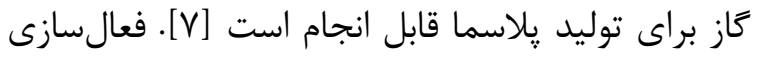

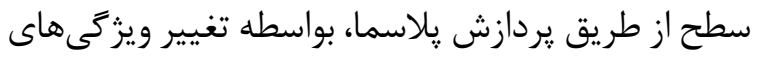

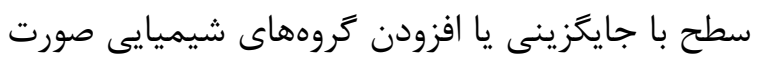

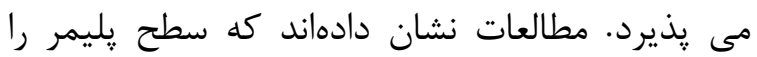

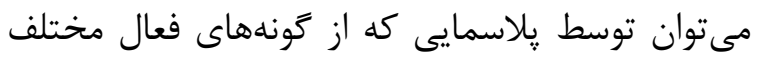

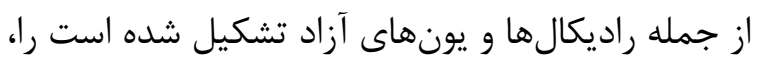

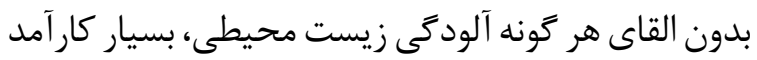

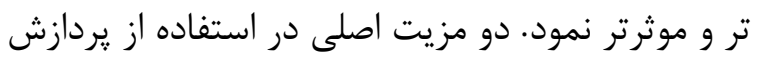

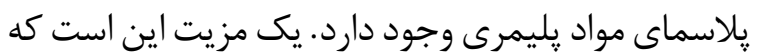

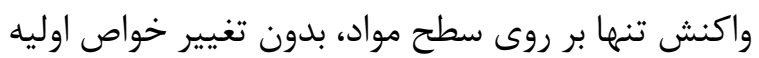

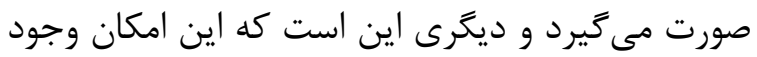

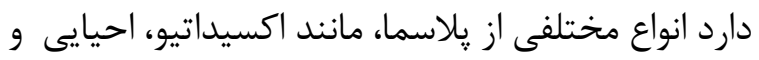

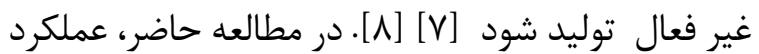

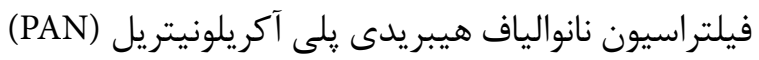

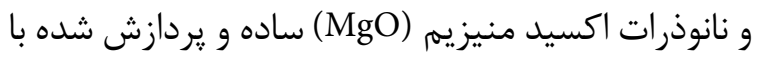

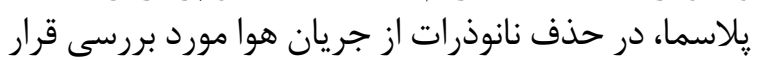

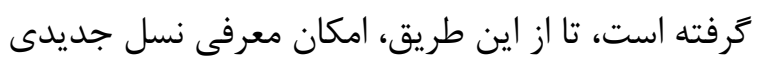

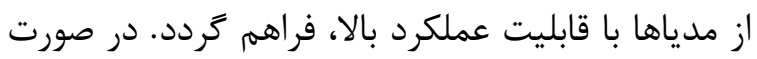

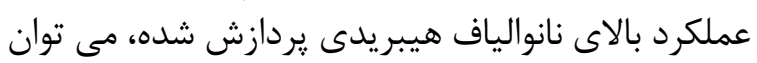

مقامها

امروزه تحقيقات كستردهاى در زمينه فناورى نانو در

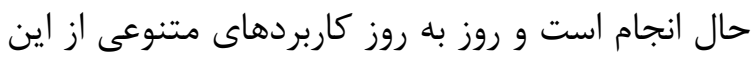

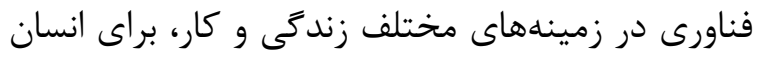

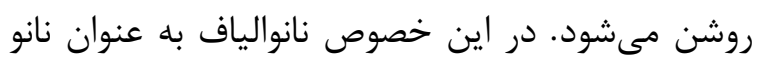

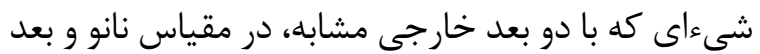

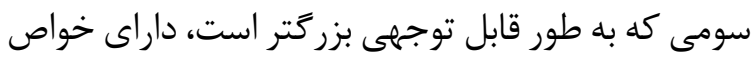

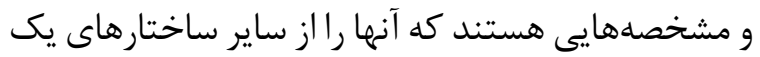

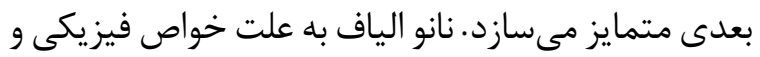

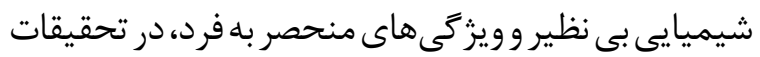

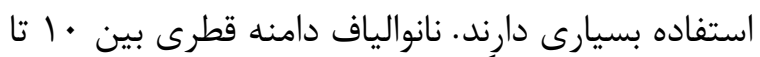

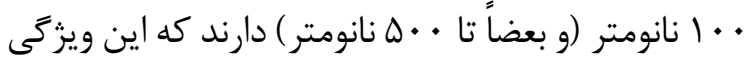

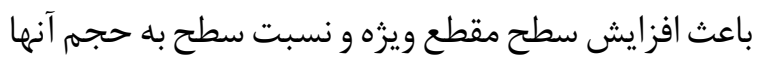

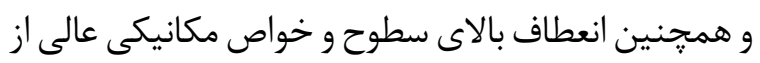

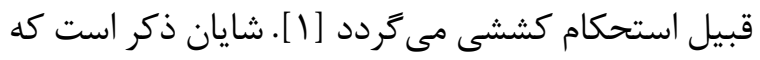

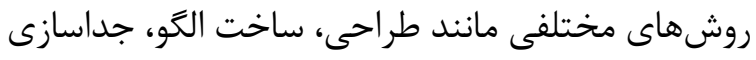

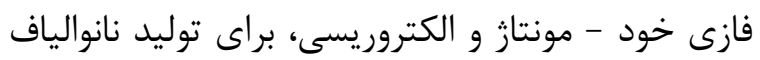

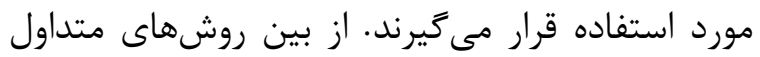

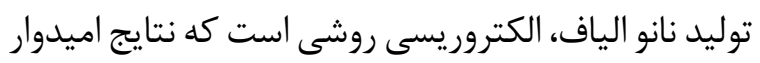

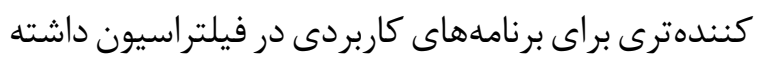

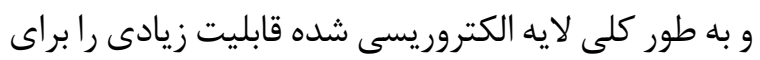

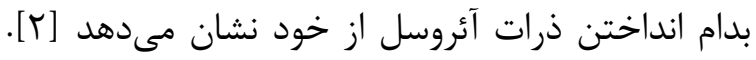

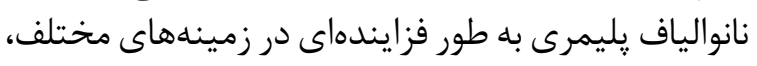

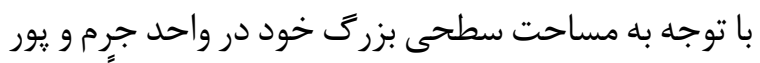

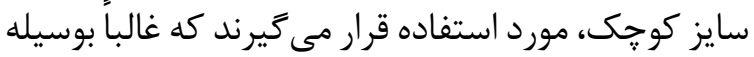

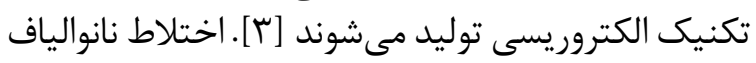
يليمرى با نانوذرات اكسيد فلزى مانند

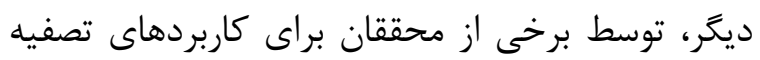

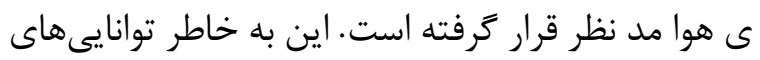

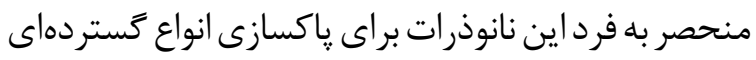

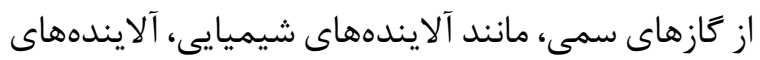

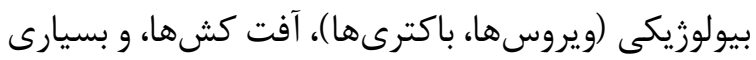

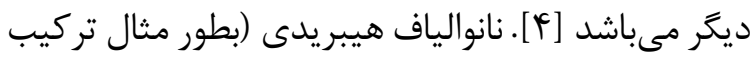

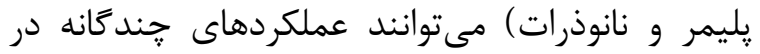

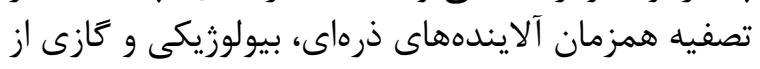

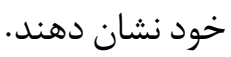
لايه نانوليفى الكتروريسى شده به به خودى خون خود نرم و

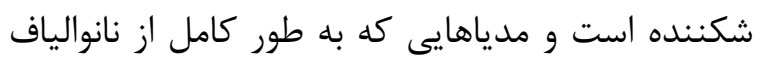

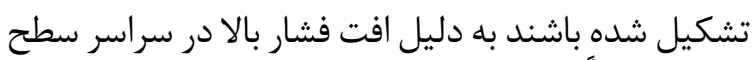

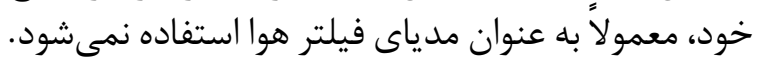

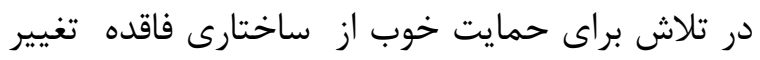

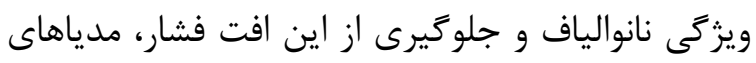


كاتد عمل مى كند. الكترود كاتد (با قطر ها هانتىمتر )

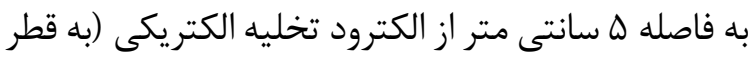

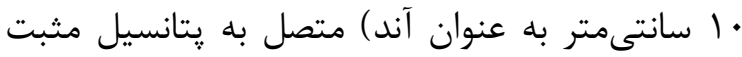

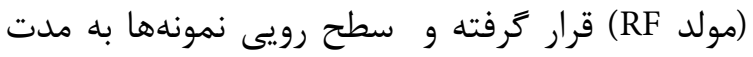

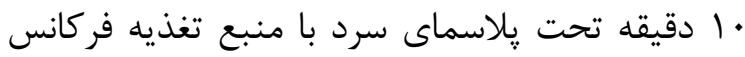

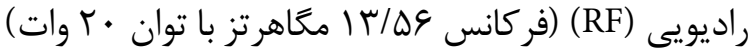

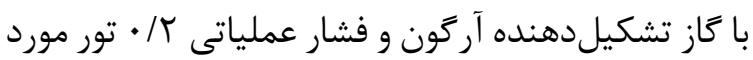

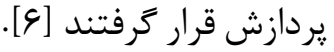

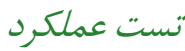

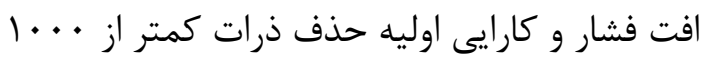

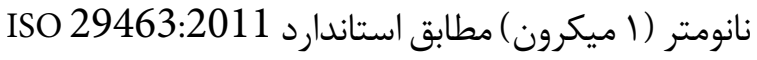

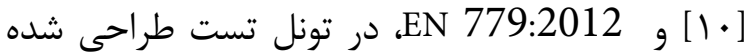

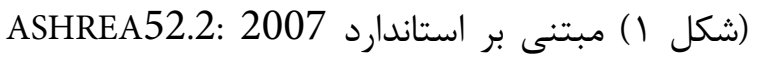

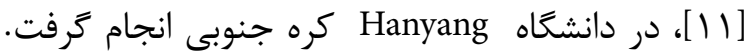

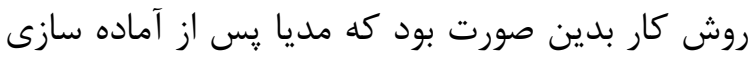

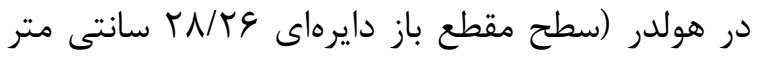

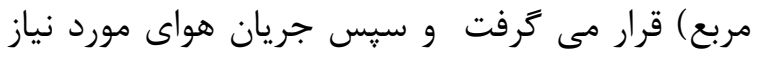

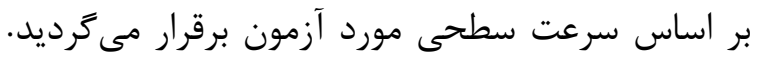

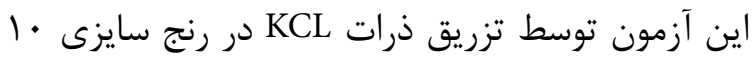

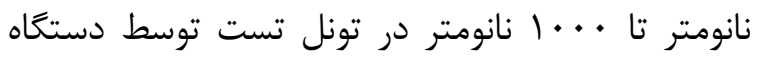

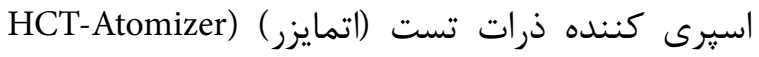
4810-South Korea و بالادست مدياى فيلتر، توسط دستخاههاى طيف ذرات سنج

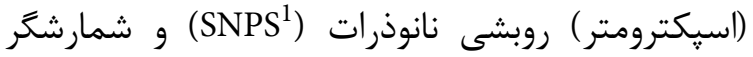

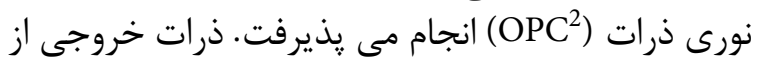

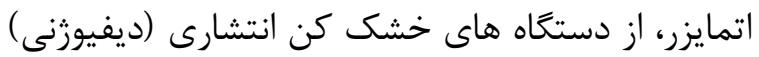
و نوترولايزر (خنثى كننده) (HCT-4920-South Korea) و به ترتيب به منظور (HCT-XRC03 -South Korea) حذف رطوبت ذرات، و خنثى شدن شارز الكتريكى ذرات

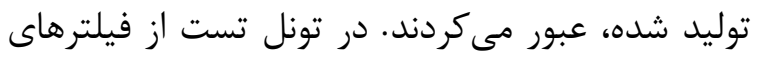

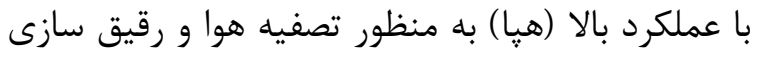

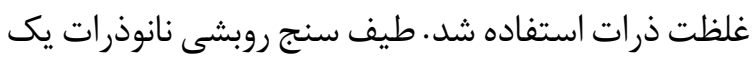

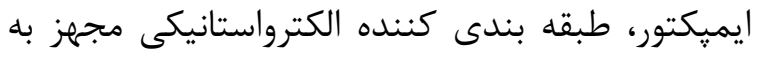

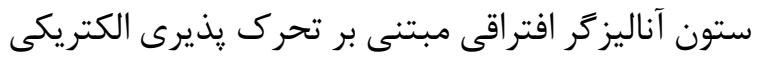

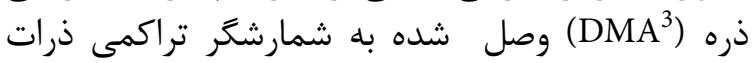
(TSI Inc., Model 3022A, USA) (CPC هواى ورودى از طريق سيستم شمارش (CNPS و (OPC) ״ايش مى گرديد تا تاثير افت فشار ايجاد شده توسط مديا

1 Scanning NanoParticle Spectrometer

2 Optical Particle Counter

3 Differential Mobility Analyzer

4 Condensation Particle Counter

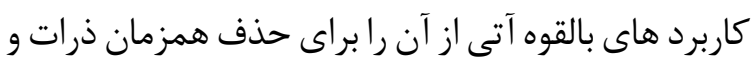

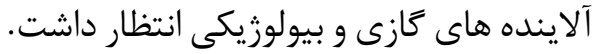

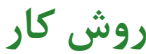

توليد نانوالياف

به منظور ساخت مدياى حاوى لايه نانوالياف، از ازئ

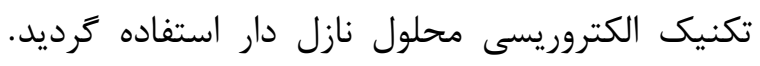

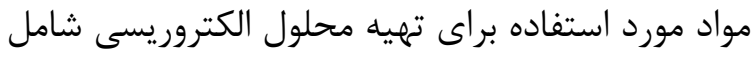

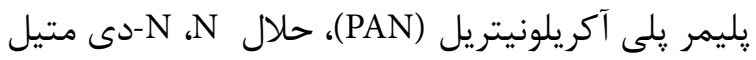

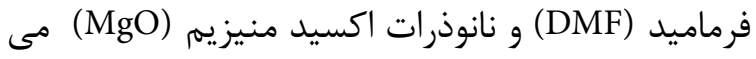

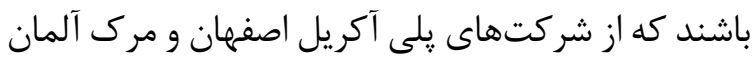
تهيه كرديدند. غلظت محلول

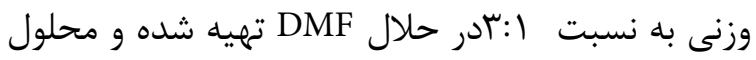

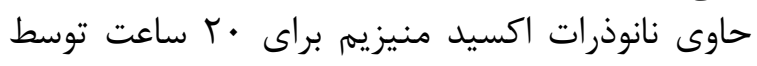

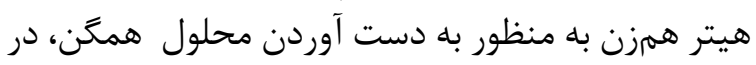

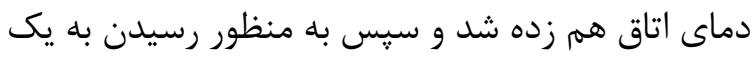

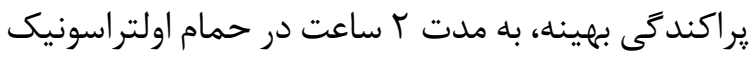

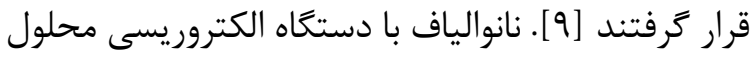

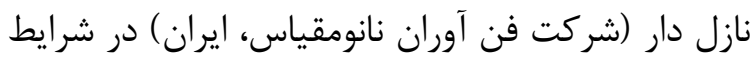

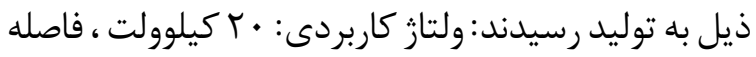

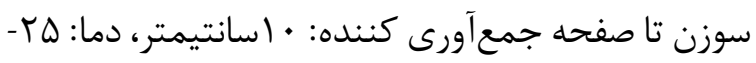

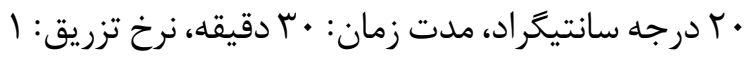

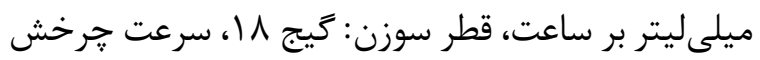

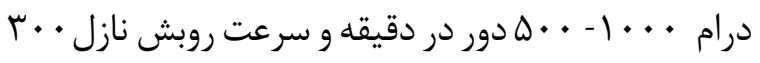

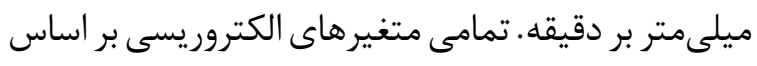

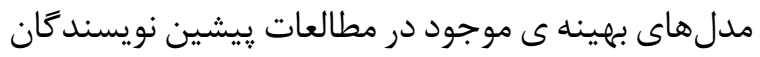

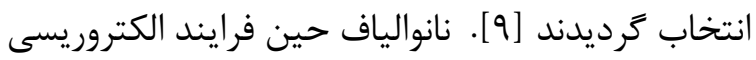

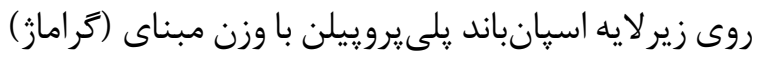
IV

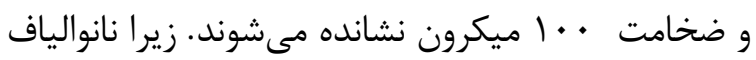

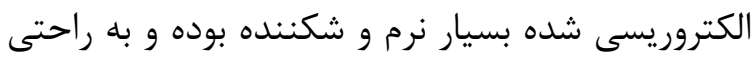

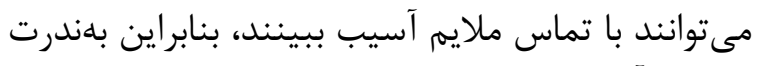

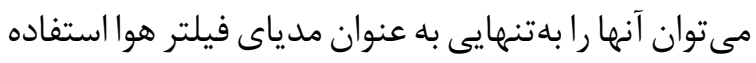

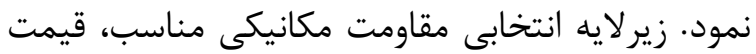

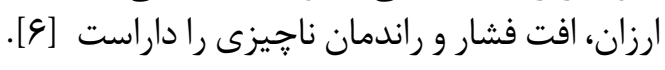

$$
\text { بردازش بلاسما }
$$

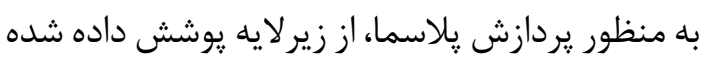

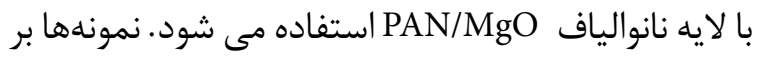

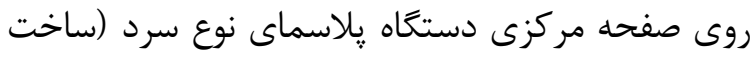

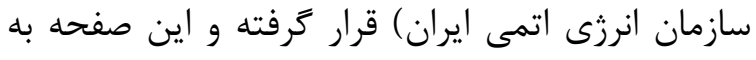

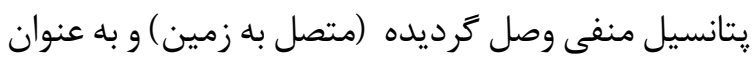




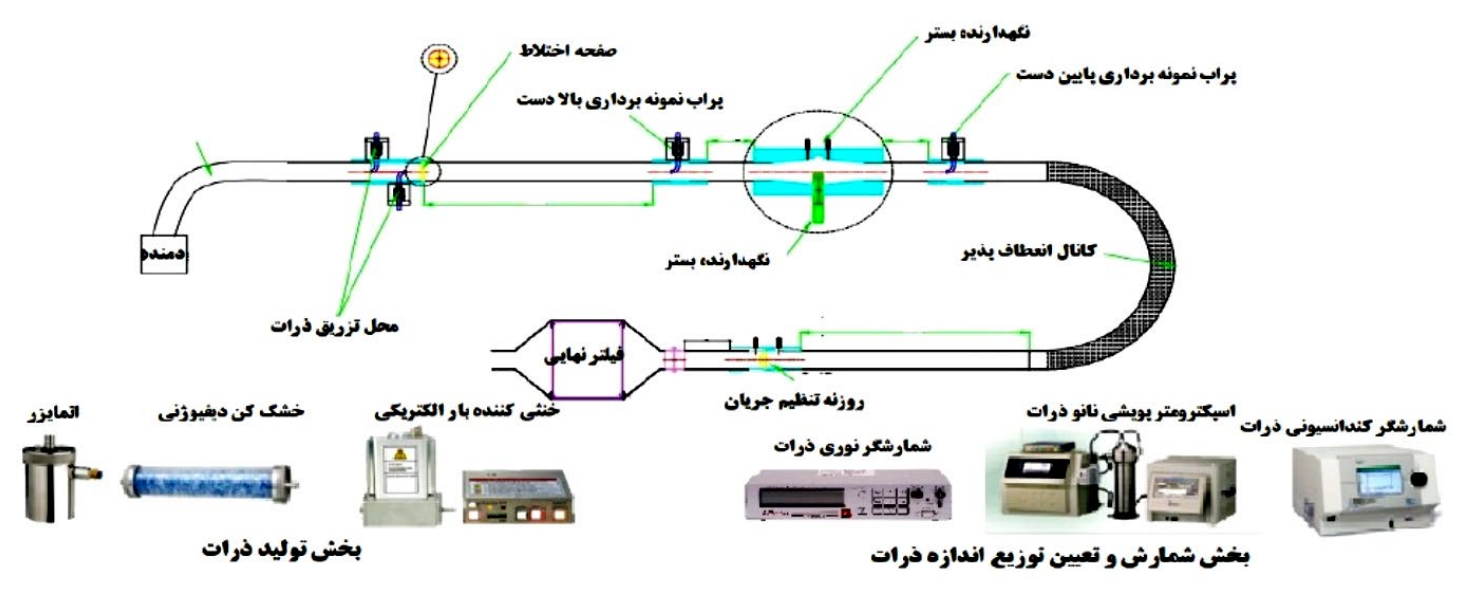

شكل ا. طرح شماتيك ست آزمون عملكرد مدياى فيلتر مورد استفاده براى ذرات زير ا ميكرون [با]

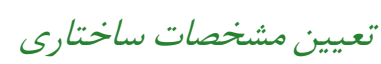

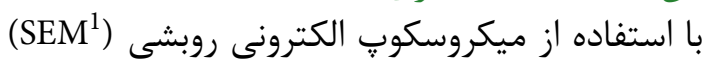
(Philips- XL 30- USA)

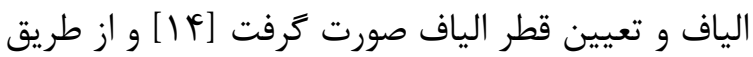
طيف سنجى تفكيك طول موج اشعه ايكس (WDX)

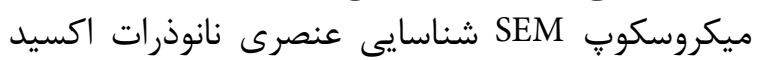

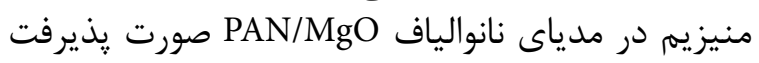

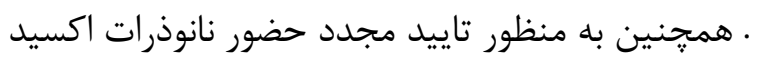

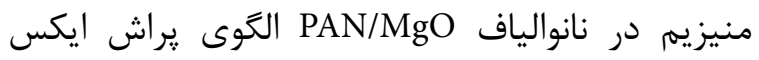
(STOE-STADIP’ Germany) (XRD³)

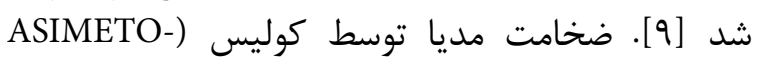
307-56-3 6" ABS- Hong Kong ازماز الخوريتمهاى تجزيه و تحليل تصاوير SEM از طريق

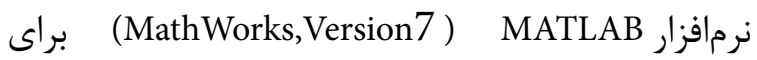
محاسبه درصد تخلخل مدياها استفاده شد [ه 1 ].

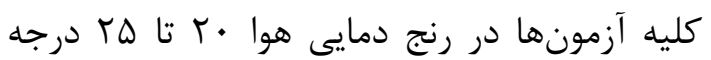

$$
\text { نتايج }
$$

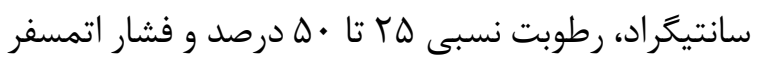

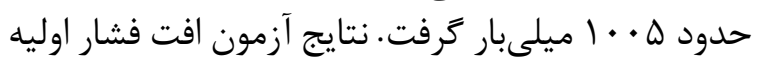

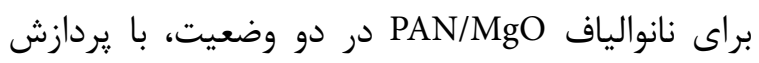

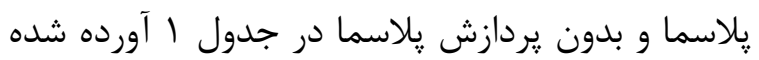

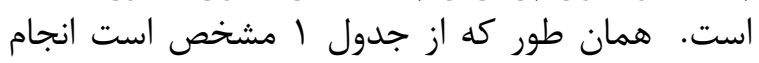

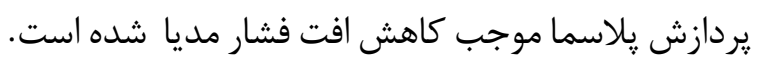

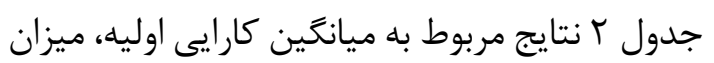

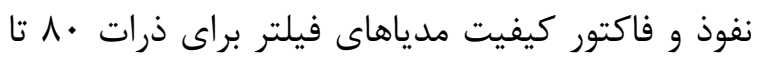
و نانومترى (مطابق استاندارد

1 Scanning Electron Microscope

2 Wavelength-dispersive X-ray spectroscopy

3 X-ray Diffraction Spectroscopy
در دبى هواى ورودى بررسى و تنظيم كردد. درجه حرارت،

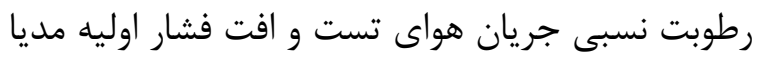

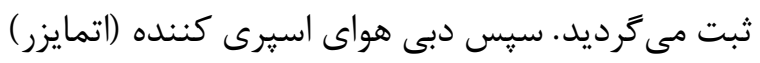

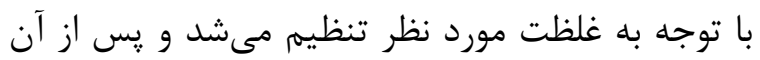

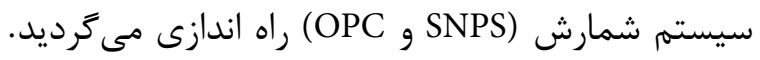

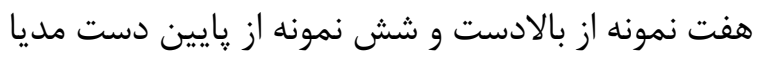

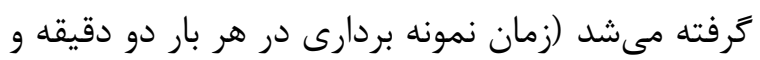

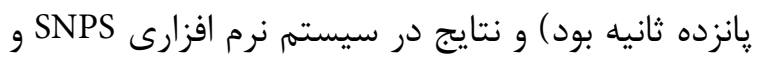

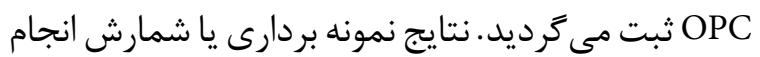

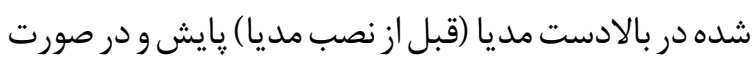

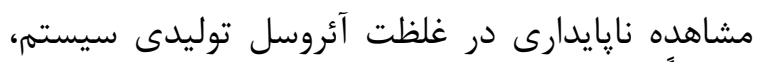

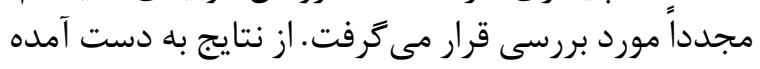

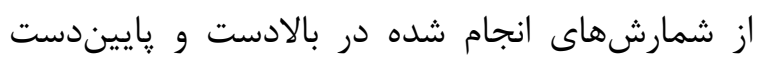

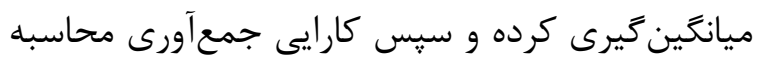

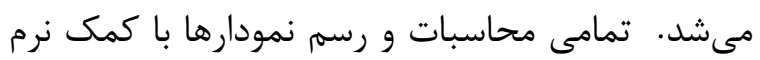

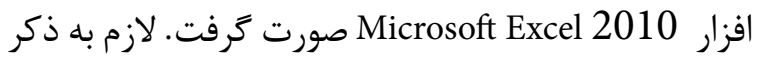

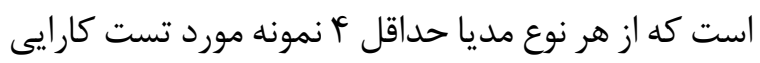
قرار كرفت [ • [].

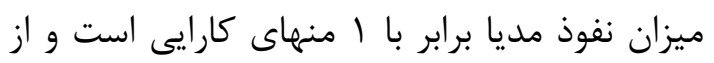

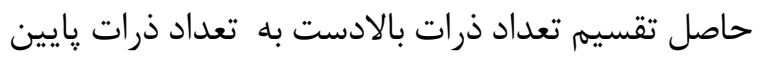

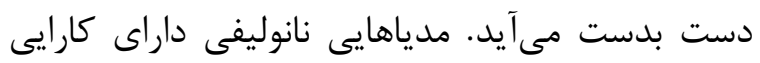

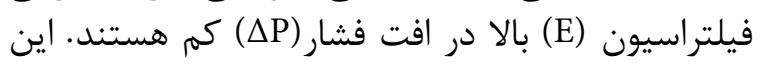

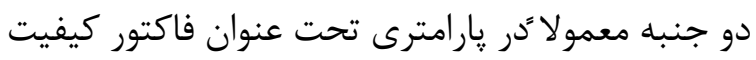

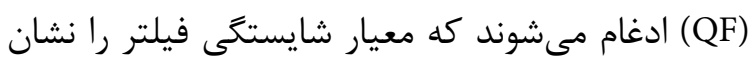

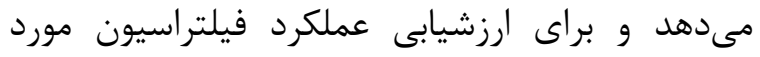

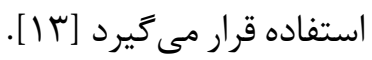

$\mathrm{QF}=-\frac{\ln (1-\mathrm{E})}{\Delta \mathrm{P}}$ 
جدول (. افت فشار اوليه مدياهاى حاوى نانوالياف PAN/ MgO (ميانكين ـ انحراف معيار)

\begin{tabular}{|c|c|c|c|c|}
\hline \multirow{2}{*}{ نوع يردازش } & \multicolumn{4}{|c|}{ افت فشار (ياسكال) بر حسب درصد كذر حجمى مورد آزمون } \\
\hline & $\Delta \cdot \%$ & $\checkmark \Delta \%$ & $1 . . \%$ & $1 r \Delta \%$ \\
\hline بدون يردازش & $F T / T \pm \cdots / 1 \Lambda$ & $\Delta \Lambda / r \pm \Psi \varepsilon / \cdot \Delta$ & $V N / r \pm T r / I I$ & $1 \cdot r / r \pm r Y / / V$ \\
\hline تحت يردازش يلاسما & $|f / \tau \pm| f / \mid$. & $|N /| \pm r \mid$ r & $r T / T \pm \cdot \cdot / r T$ & $r N / I \pm V Y / \cdot q$ \\
\hline
\end{tabular}

جدول r. نتايج عملكرد مدياهاى حاوى نانوالياف PAN/ MgO براى حذف ذرات زير ا ميكرون

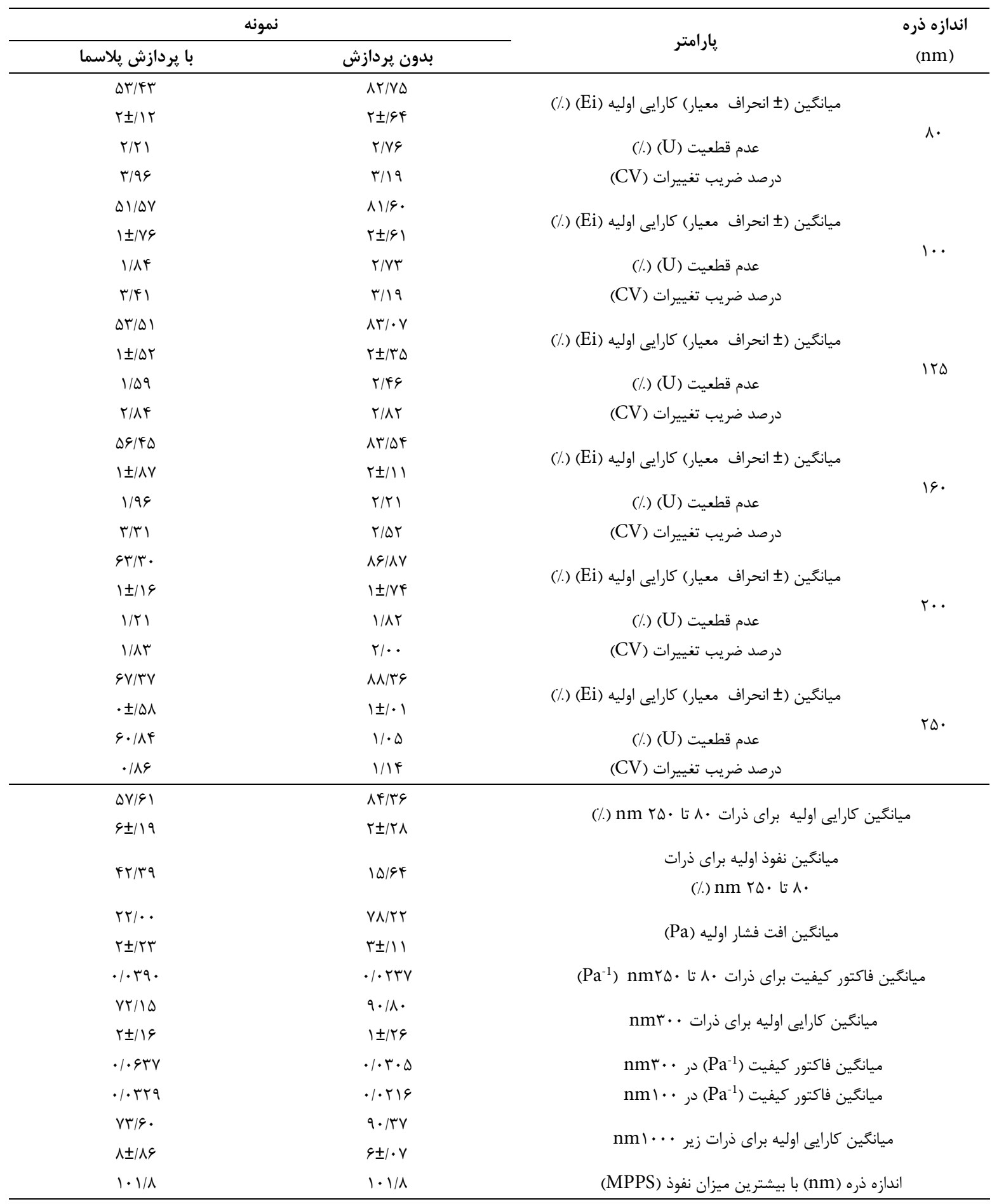


منحنى عملكرد فيلتراسيون زيرلايه و مقايسه كارايى

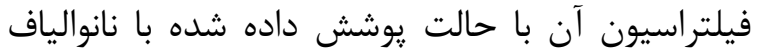

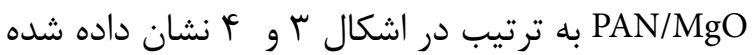

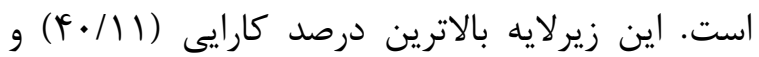

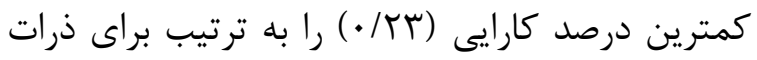

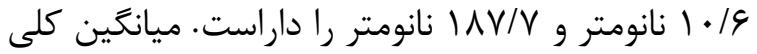

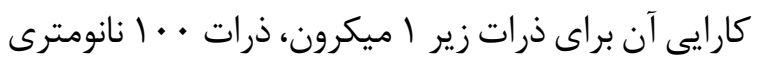

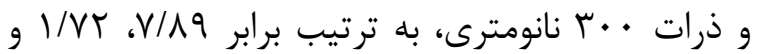

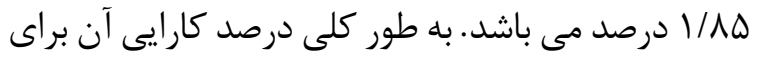

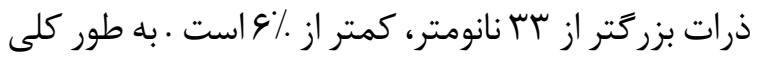

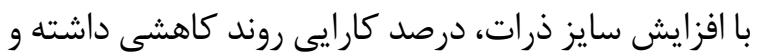

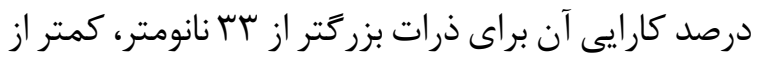

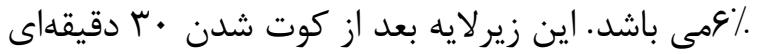

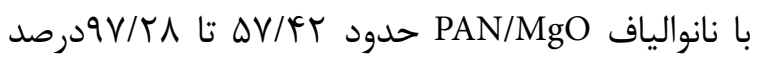
افزايش كارايى فيلتراسيون را از خود نشان داده داف و ديكر

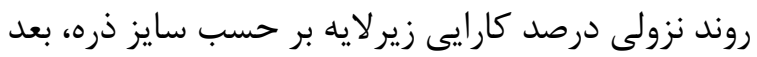

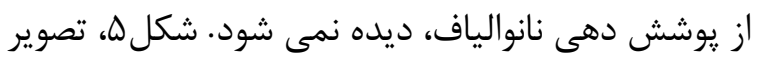

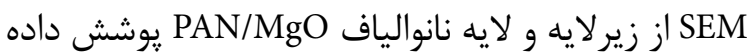

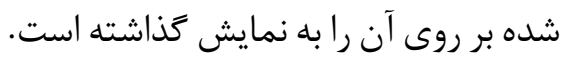

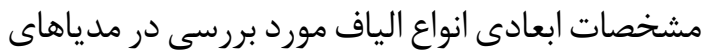

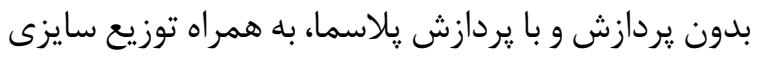

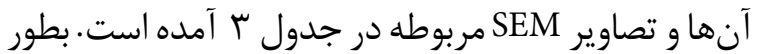
كلى نانوالياف PAN/MgO ساده از قطر الياف كوجناون

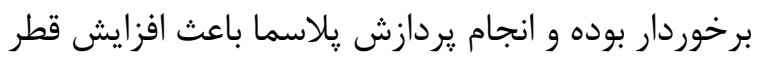

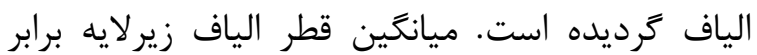
(

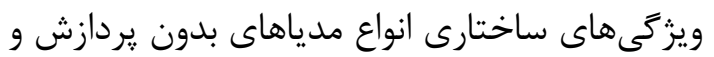

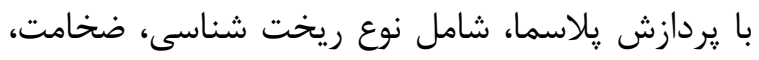

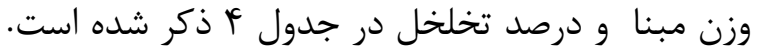

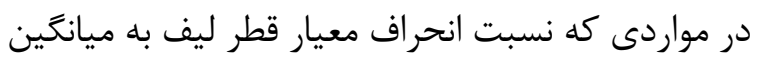

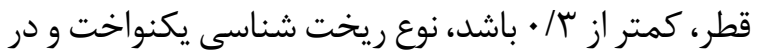

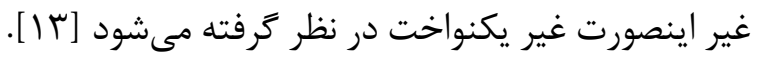

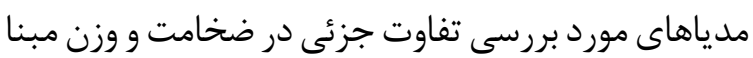

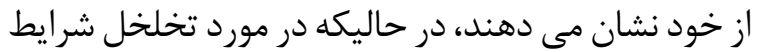
متفاوتى داشتند.

روش طيف سنجى تفكيك طول موج اشعه ايكس

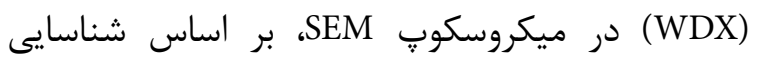
عنصرى نانوذرات اكسيد منيزيم در مدياى نانوالياف / عندئ

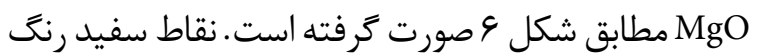

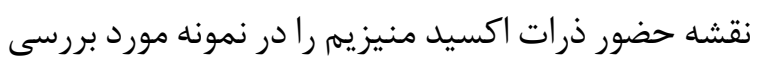

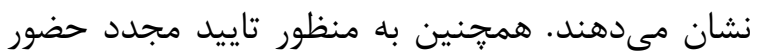

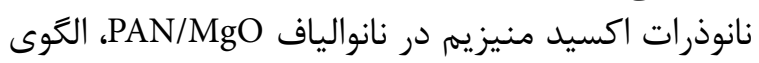

همجنين ميانگين كارايى كل براى ذرات زير ا ميكرون

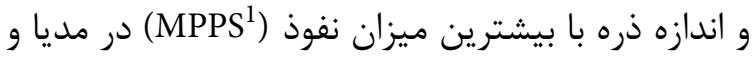

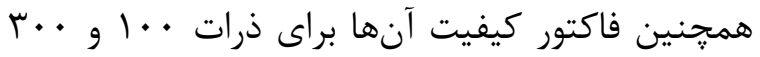

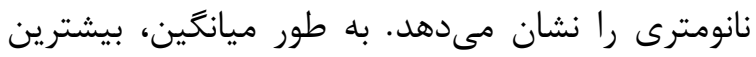

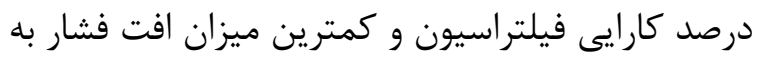

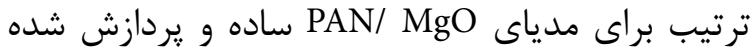

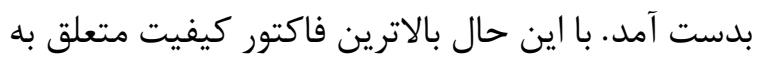

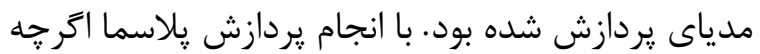

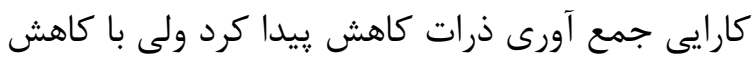

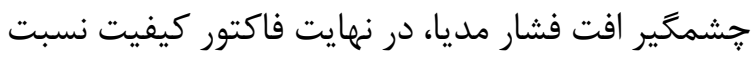

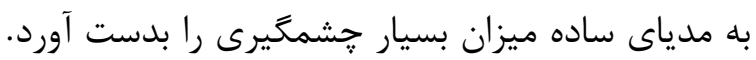

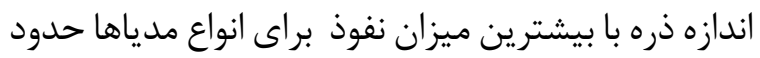

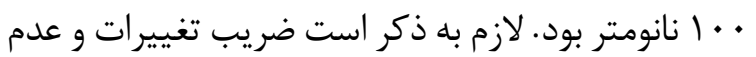

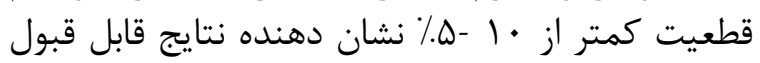

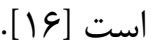

شكل r ميانكين كارايى و فاكتور كيفيت مدياهاى

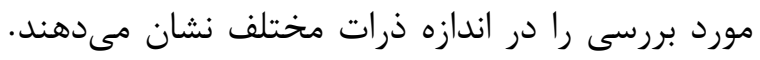

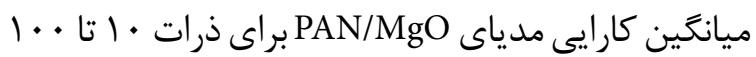

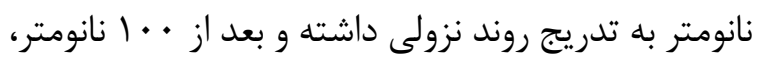

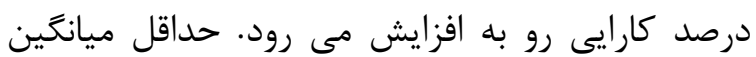

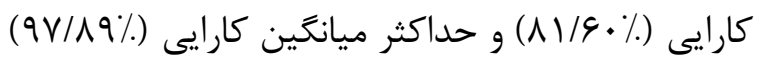

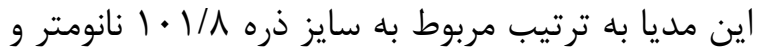

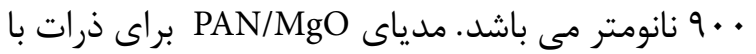

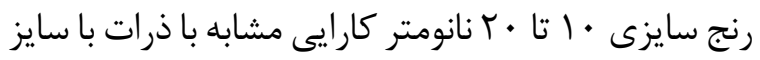

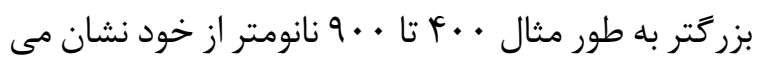

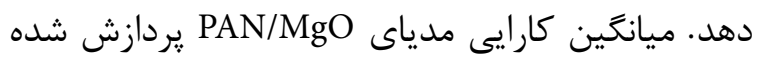

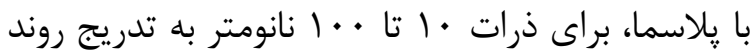

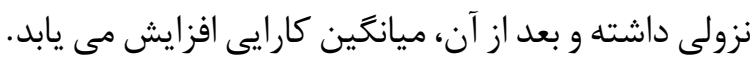

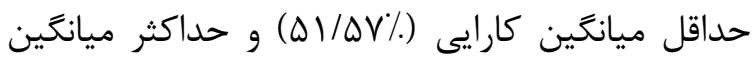

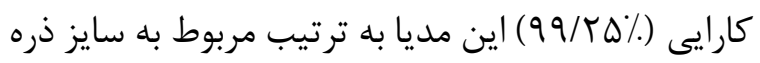

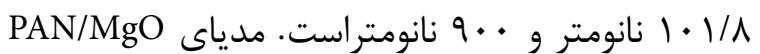

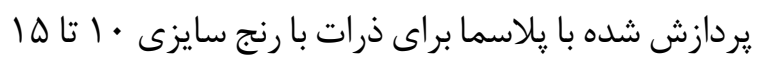

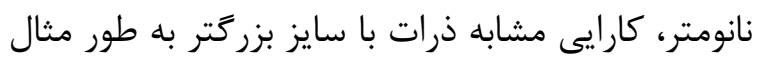

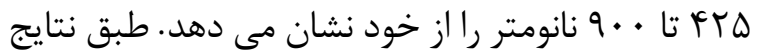

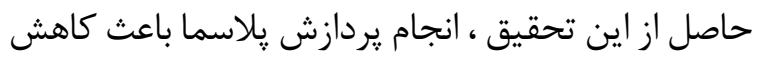

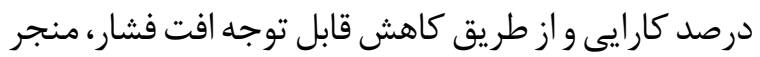

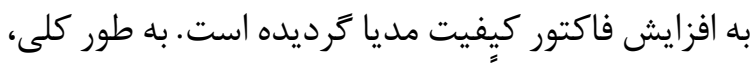

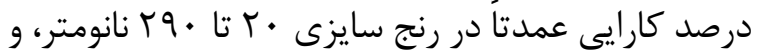

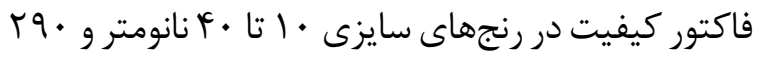

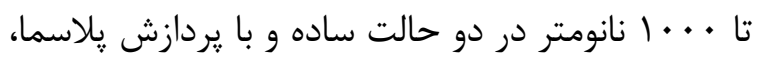
داراى اختلاف فاحشترى مي داشن داند. 

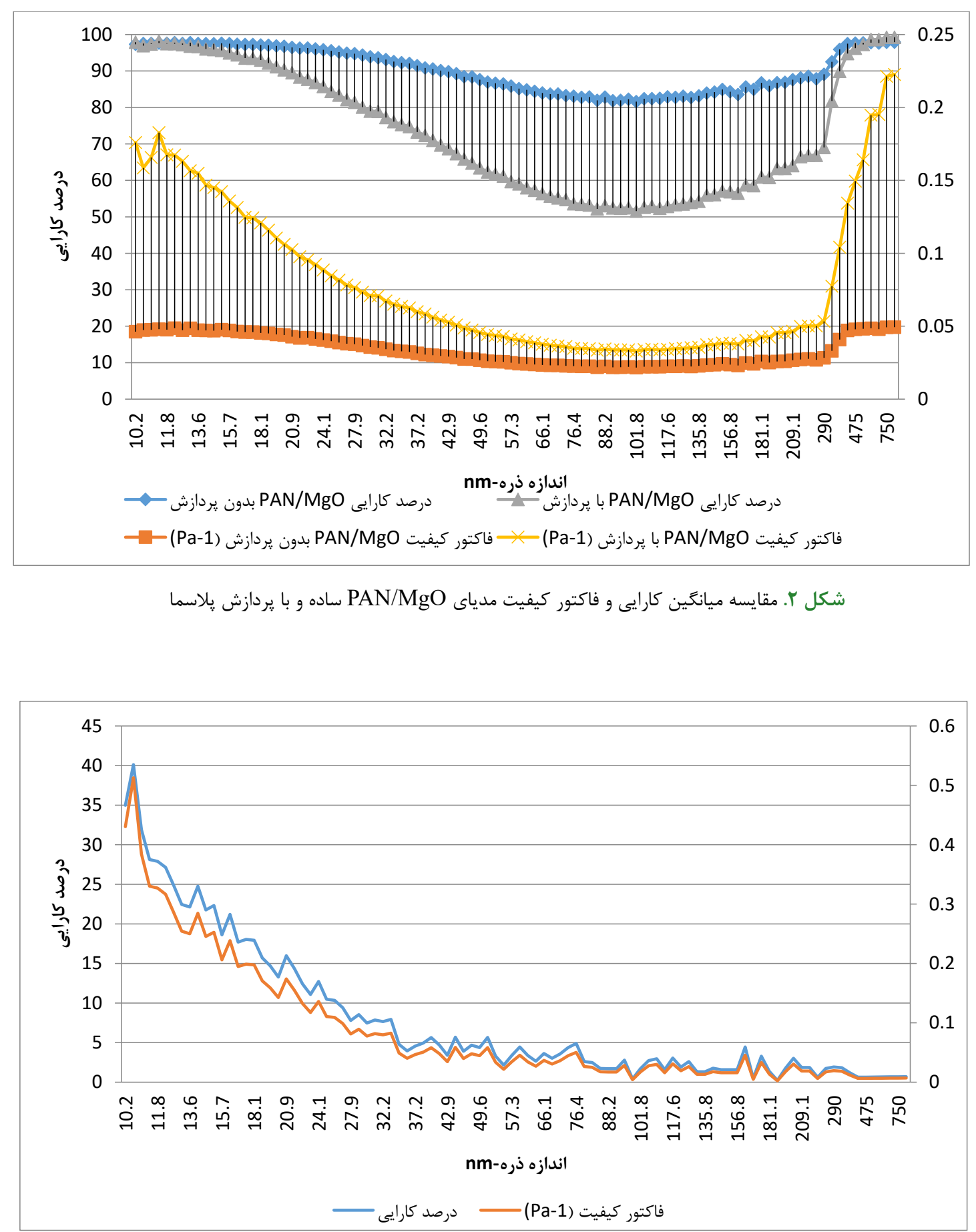

شكل r. منحنى عملكرد فيلتراسيون زيرلايه (افت فشار= (ياسكال)

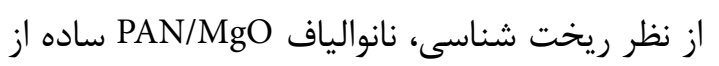

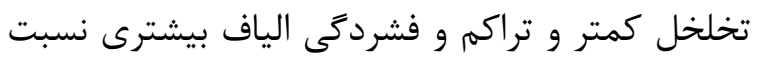

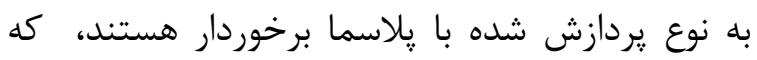

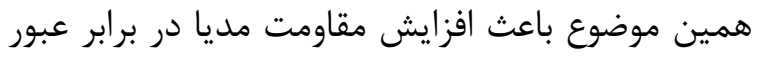

يراش ايكس (XRD) نيز از آن تهيه شده است (شكل V).

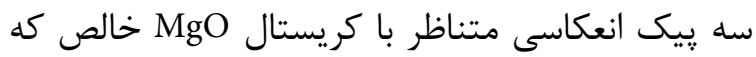

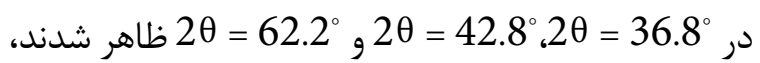
نشان مى ندهند كه نانوالياف الكتروريسى شده حاوى كريستال MgO خالص مى باشند [IV] 
مقايسه عملكرد فيلتراسيون نانوالياف PAN/MgO ساده و يردازش شده با پلاسما ...

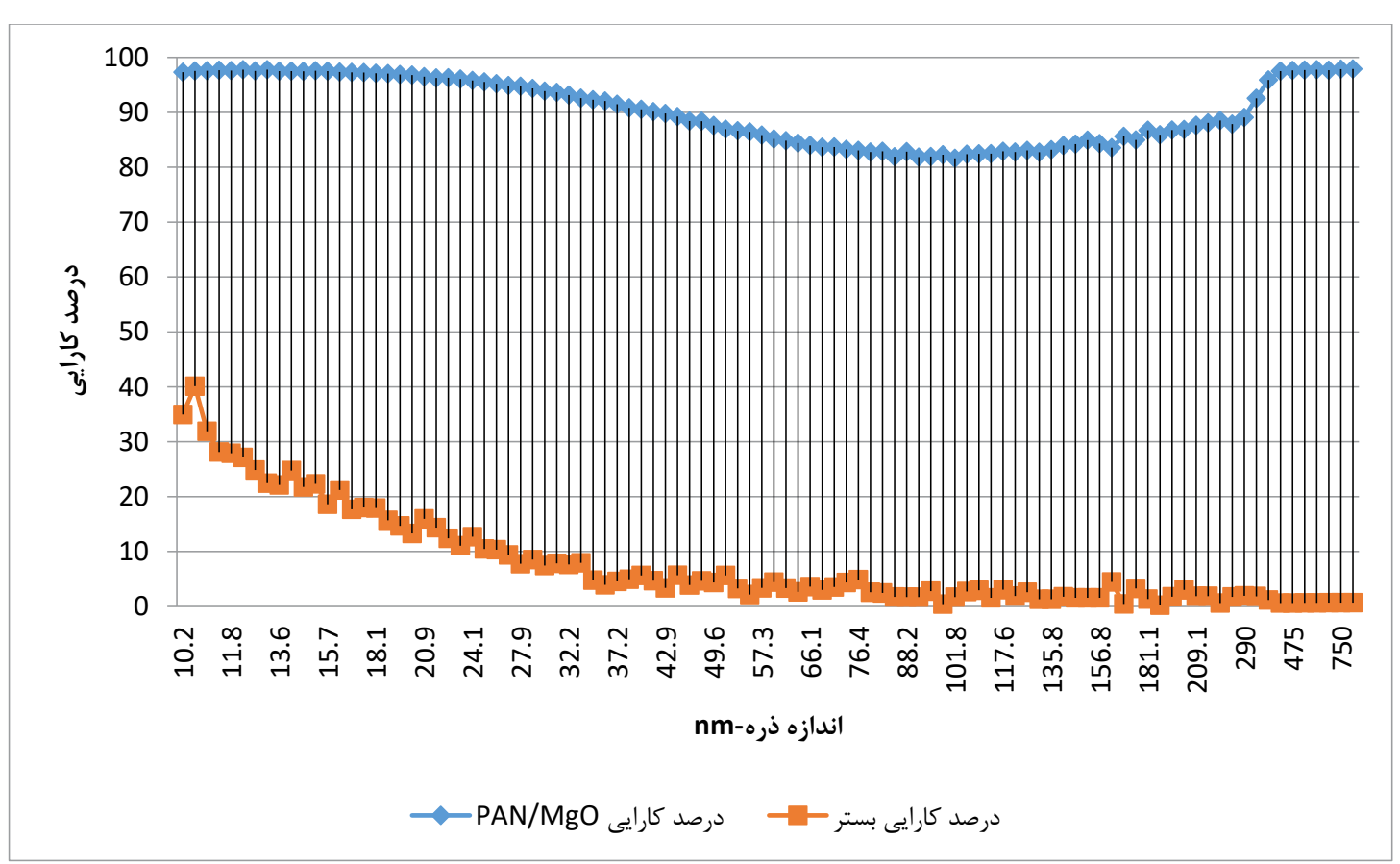

شكل F. مقايسه كارايى فيلتراسيون زيرلايه (بستر) و زيرلايه يوشش داده شده با نانوالياف PAN/MgO

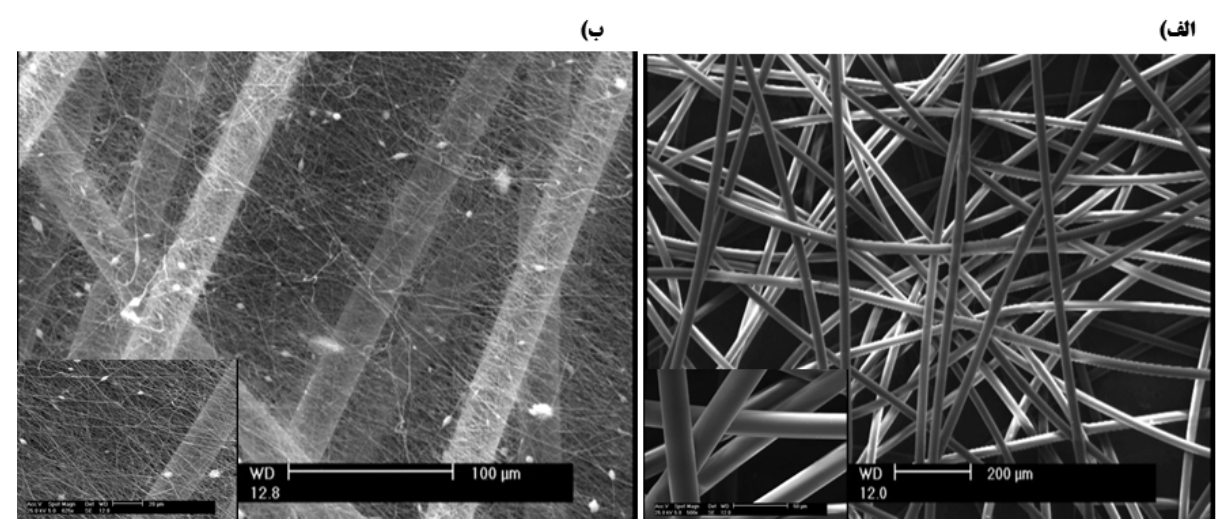

شكل ه. تصوير SEM از زيرلايه (الف) و لايه نانوالياف PAN/MgOيوشش داده شده بر روى آن (ب)

در رزيمم لغزشى ديده مىشود [1 [1].اين موضوع به خوبى

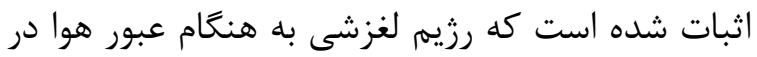

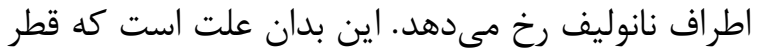

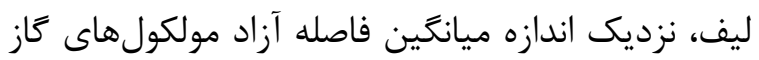

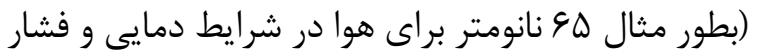

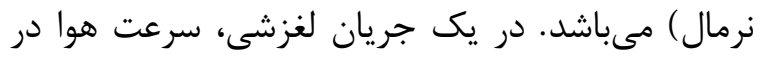

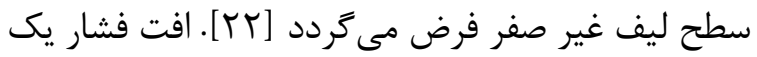
مدياى فيلتر، نسبت به به قطر حفره بسيار حساس است،

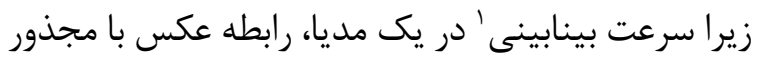
1 Interstitial velocity

in
هوا، كاهش نفوذيذيرى آن و افزايش افت فشار شده

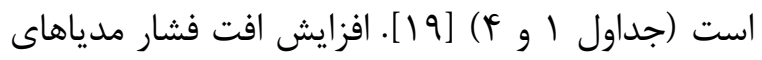
حاوى نانوالياف PAN/MgO ساده نسبت به يردازش شده

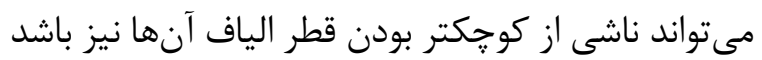
(جدول ب). طبق تئورى كلاسيك فيلتراسيون، افت فئار

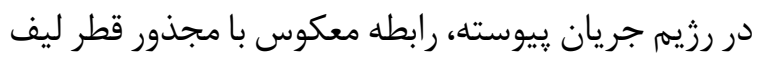

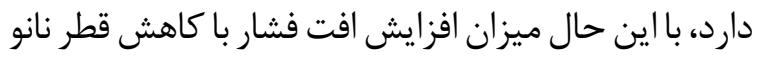

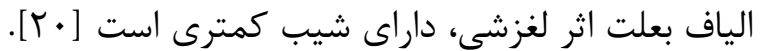

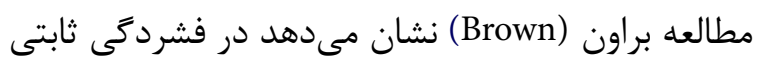

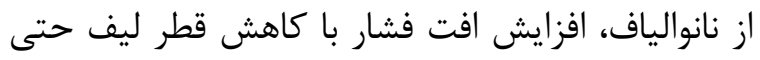
Iran Occupational Health. 2021 (01 Feb);18: 4 
جدول س. مشخصات ابعادى نانوالياف PAN/MgO ساده و با يردازش يلاسما

PAN/MgO

جدول f. مشخصات ساختارى انواع مدياى بدون يردازش و با يردازش يلاسما

\begin{tabular}{|c|c|c|c|c|}
\hline (درصد) & (ترم بر مترمربع مبنا مديا) & 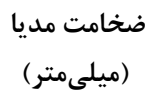 & ريخت شناسى & مديا \\
\hline il & $\mid V / \Delta \Lambda$ & .1110 & دانه دار" & ساده \\
\hline is & $\mid V / \Delta \Lambda$ & .1110 & دانه دار & يردازش يلاسما \\
\hline
\end{tabular}

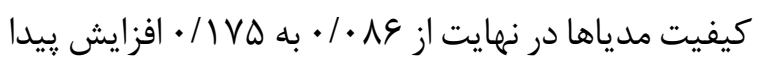

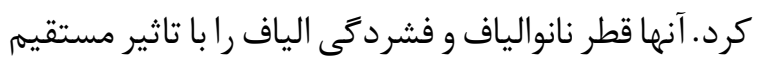

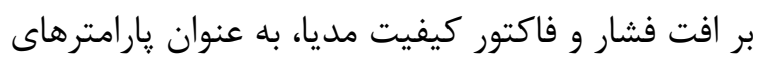

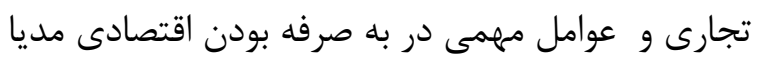

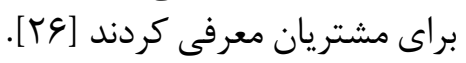

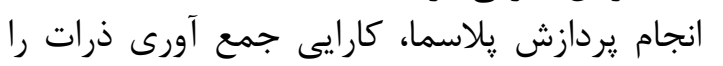

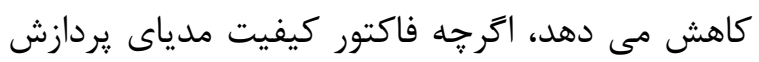

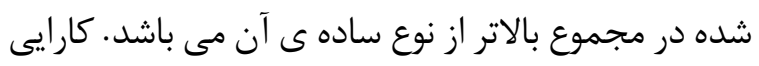

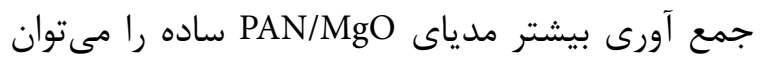

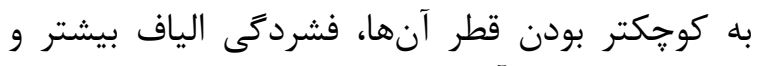

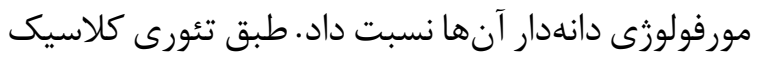

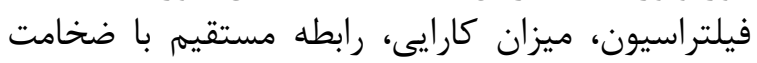

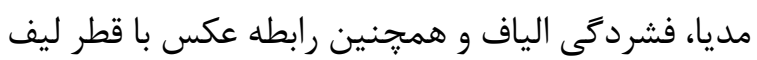

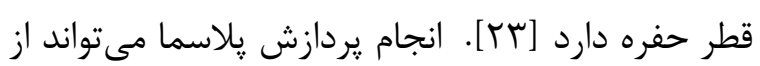

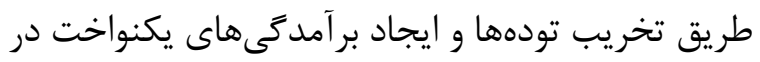

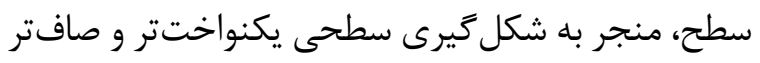

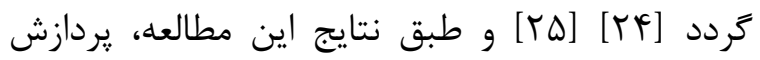

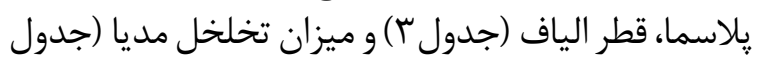

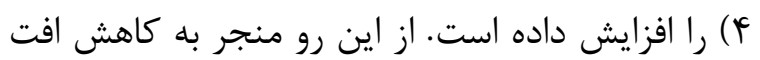

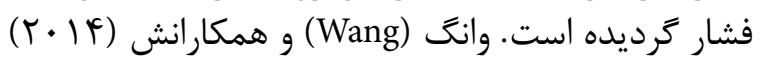

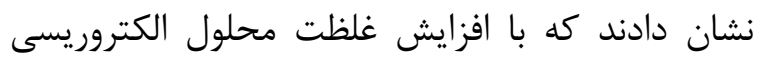

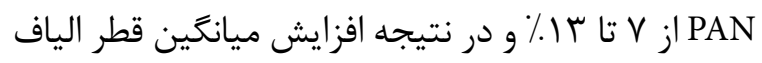

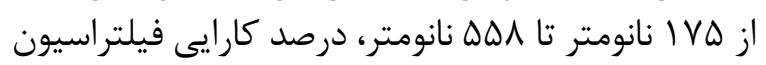

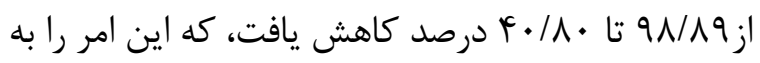

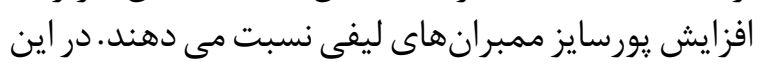

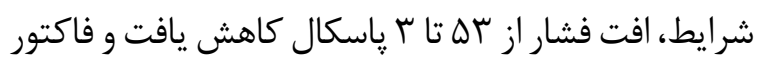




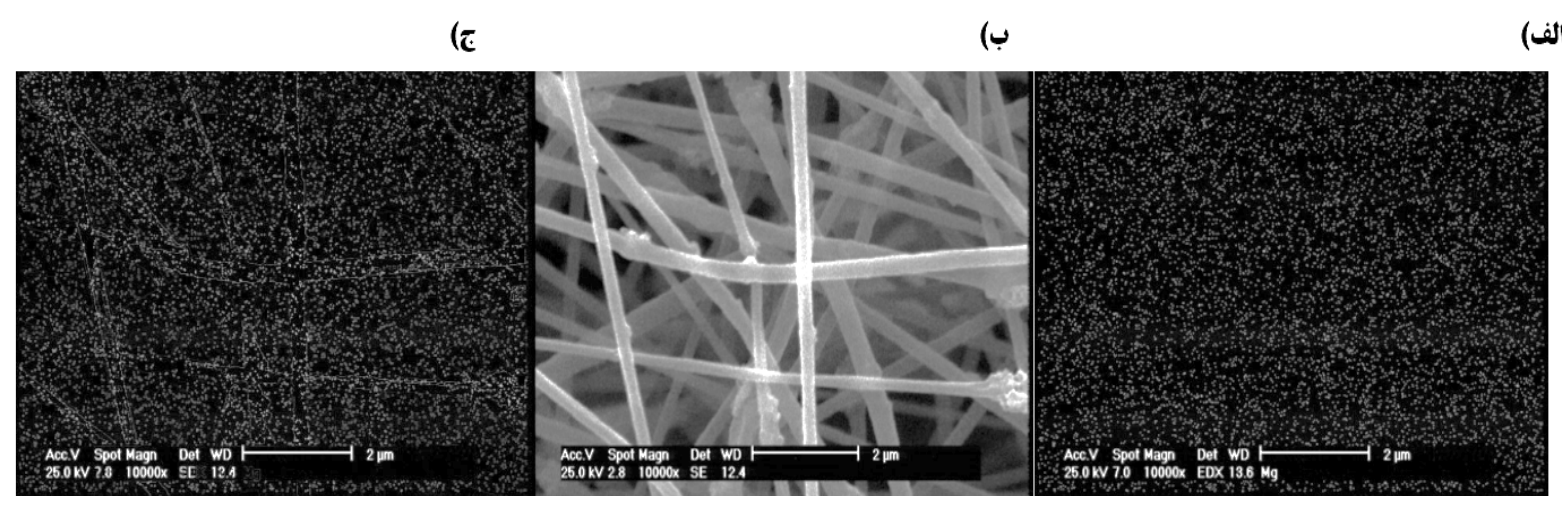

شكل و. نقشه عنصرى نانوذرات اكسيد منيزيم (الف)، تصوير SEM نانوالياف PAN/MgO (ب) و نقشه عنصرى نانوذرات اكسيد منيزيم به همراه

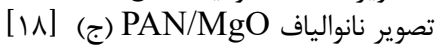

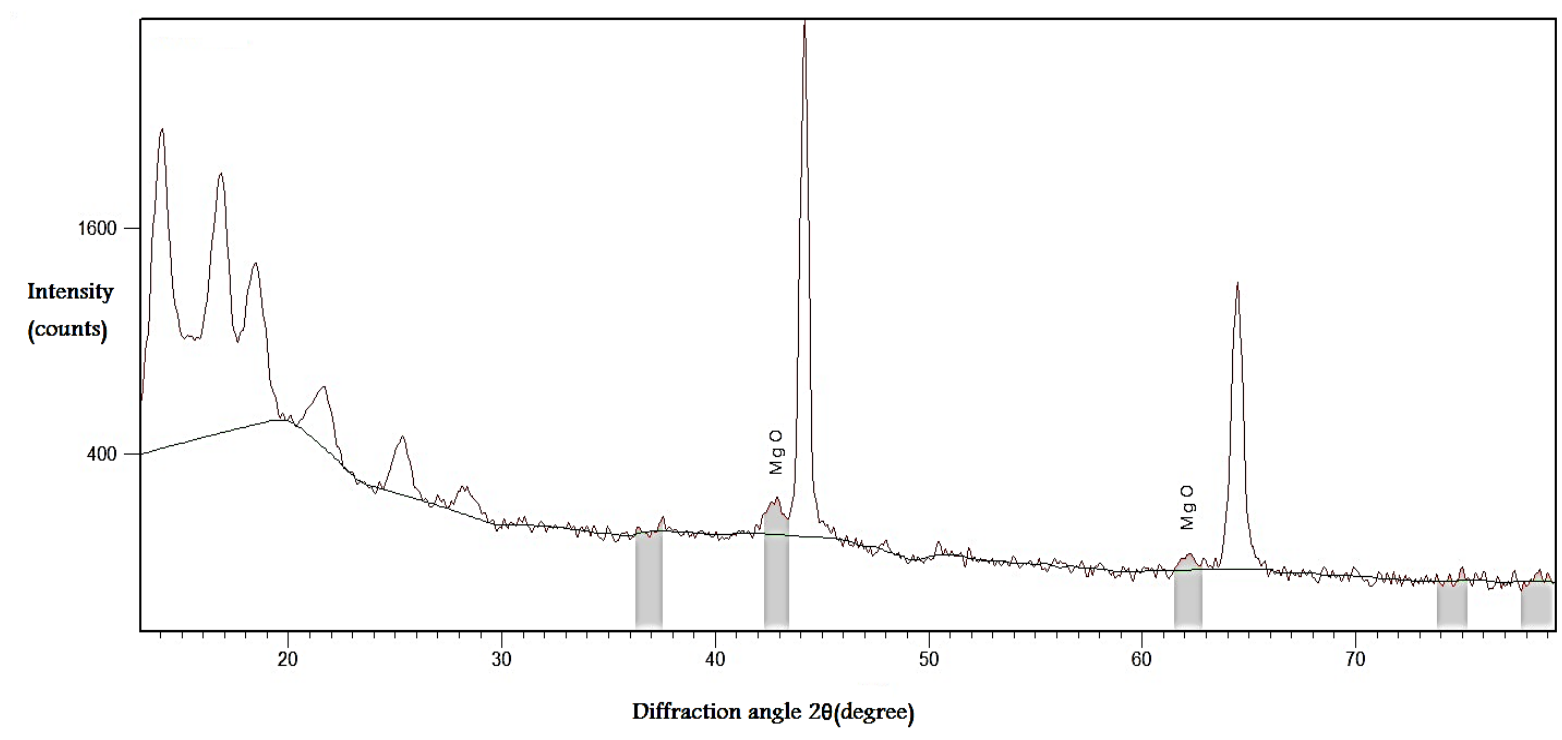

شكل V. الكَوى براش ايكس (XRD) مدياى حاوى نانوالياف PAN/MgO) مل

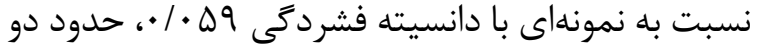

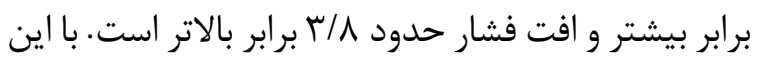

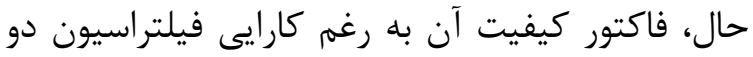

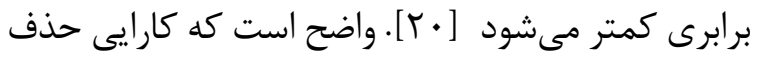

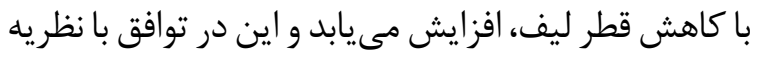

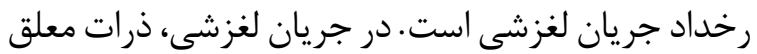

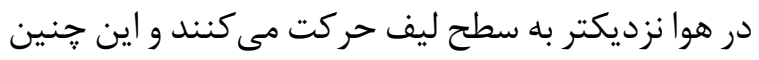

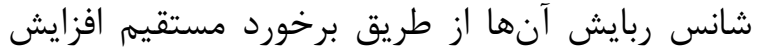

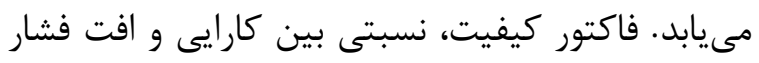

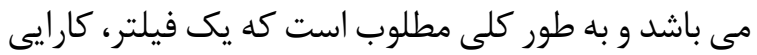

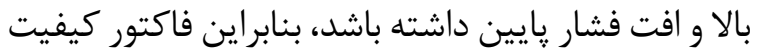

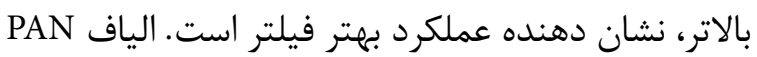

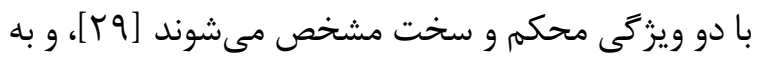

و ميزان تخلخل دارد [•[ب]. الياف با قطر كوجكتر داراى مساحت رويه بيشتر، تراكم و جُمالى بالاتر و سايز حفره

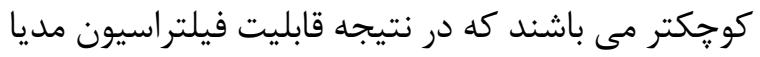

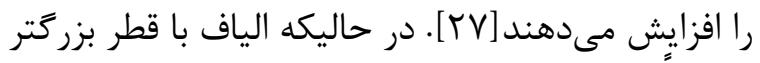

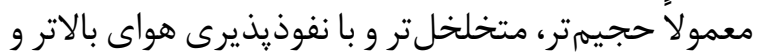
افت فشار كمترى همراه هستند [Y [Y].

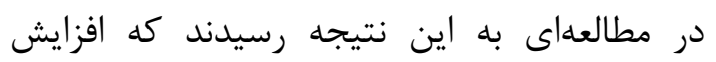

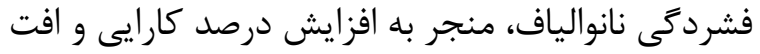

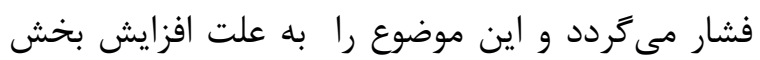

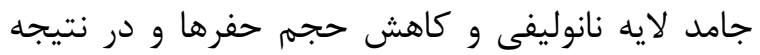

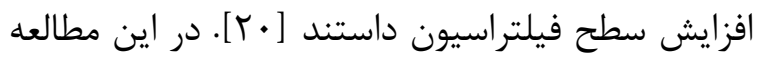

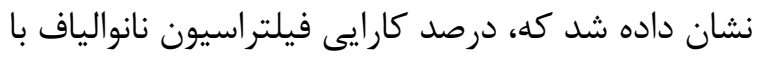

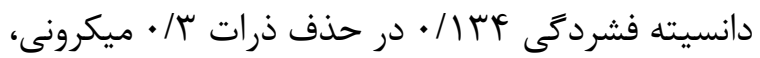


اترجه كارايى جمع آورى ذرات كاهش بيدا كرد، ولى با

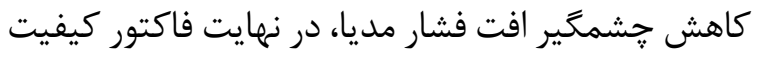

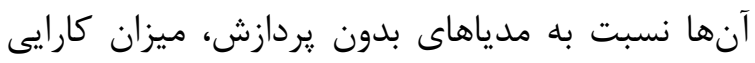

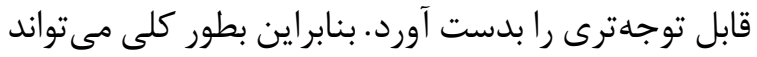

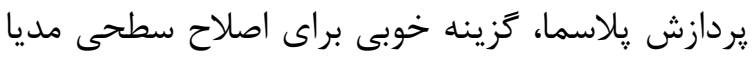

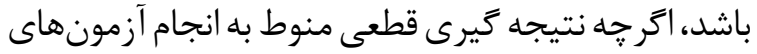

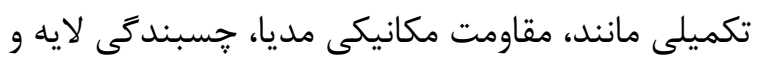

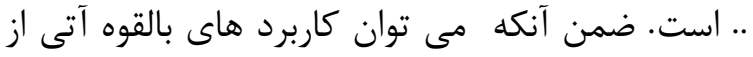

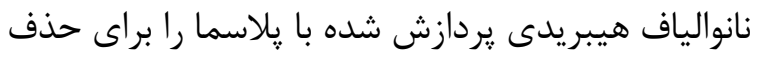

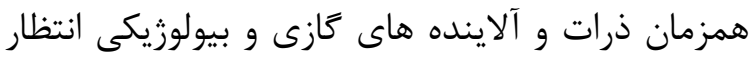
داشت. يردازش يلاسما باعث افزايش قطر الياف و در نتيجه إنها

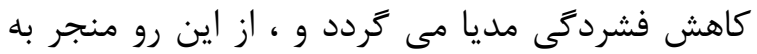

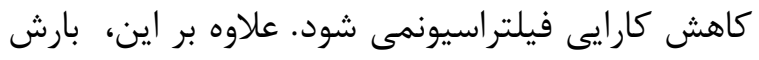

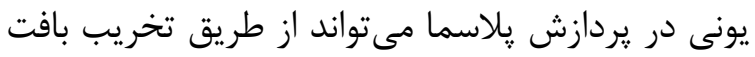

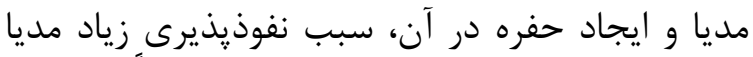

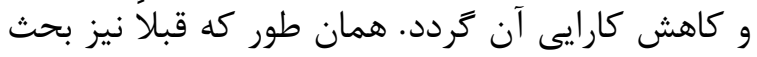

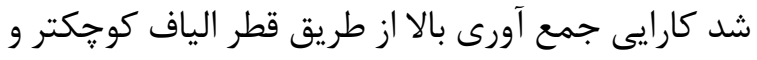

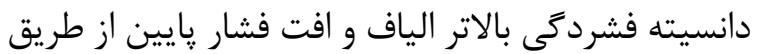

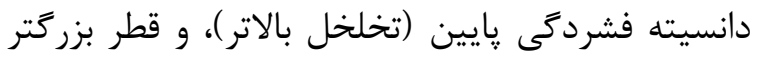

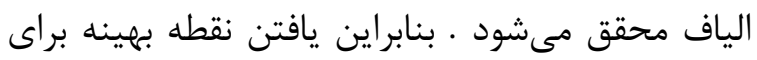

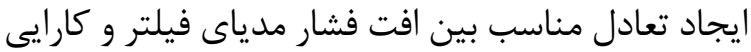

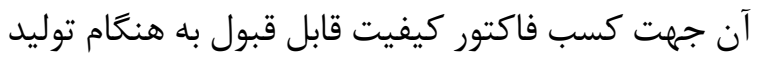

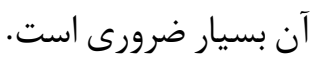

$$
\text { تشكر و قدردانى }
$$

اين مقاله حاصل بخشى از طرح تحقيقاتى مصوب

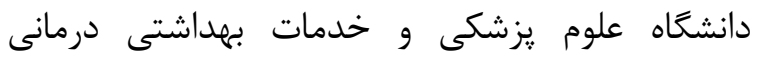

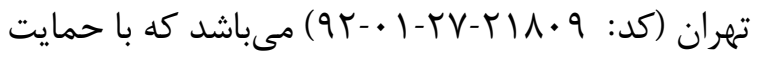

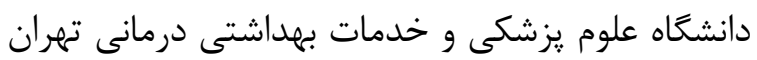

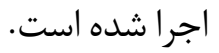

\section{منابع}

1. Kenry, Lim CT: Nanofiber technology: current status and emerging developments. Progress in Polymer Science 2017, 70:1-17.

2. Wang G, Yu D, Kelkar AD, Zhang L: Electrospun nanofiber: Emerging reinforcing filler in polymer matrix composite materials. Progress in Polymer Science 2017, 75:73-107.

3. Tao D, Wei Q, Cai Y, Xu Q, Sun L: Functionalization of polyamide 6 nanofibers by electroless deposition of copper. Journal of Coatings Technology and Research 2008, 5(3):399-403.

4. Balamurugan R, Sundarrajan S, Ramakrishna S: Recent
همين دليل است كه مىتوانند زَينه خوبى براى كاربرد

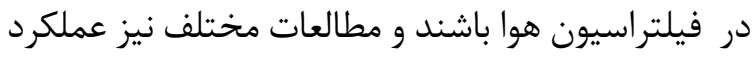

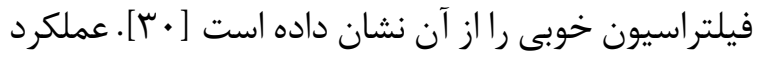

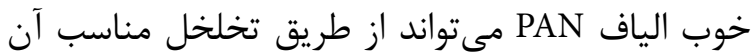

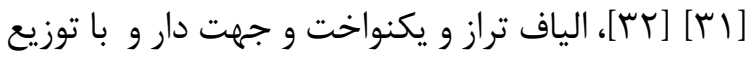

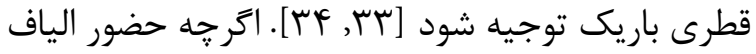

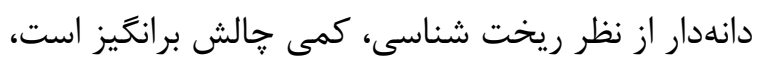

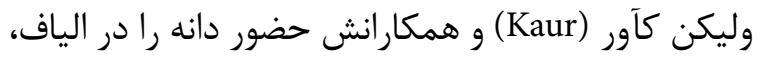

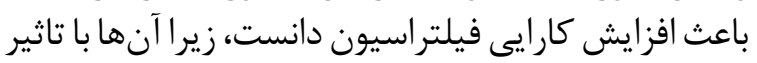

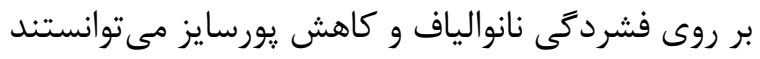

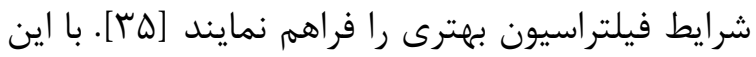

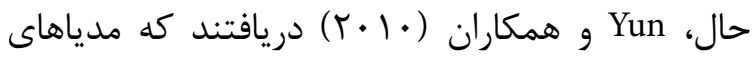

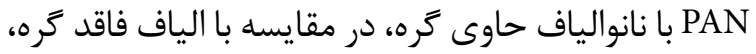

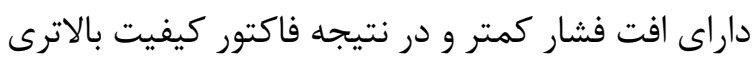

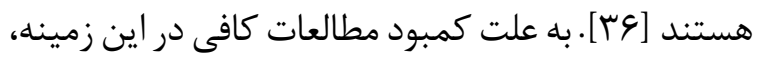

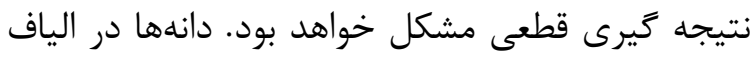

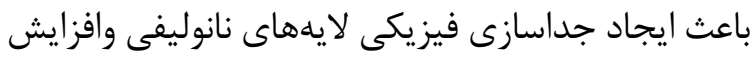

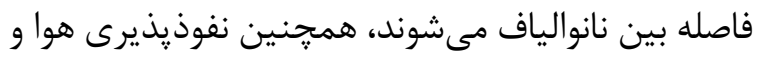

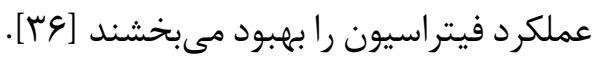

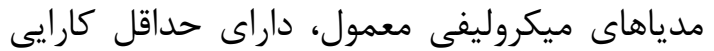

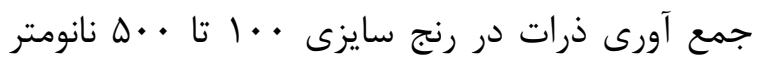

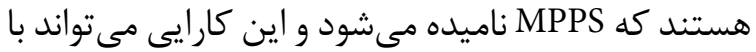

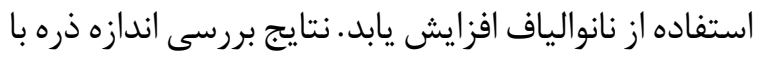

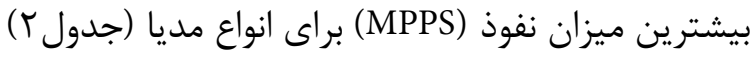

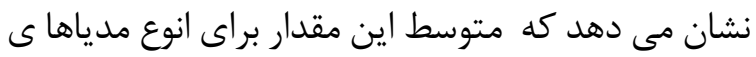

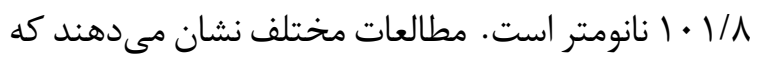

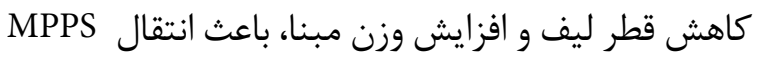

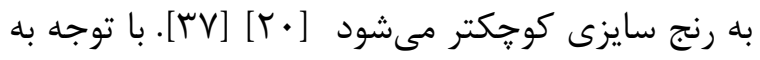

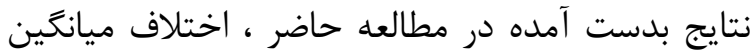

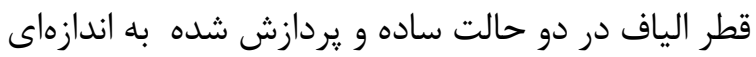

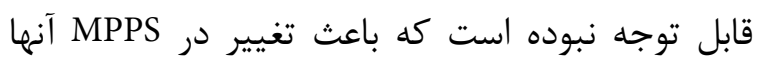

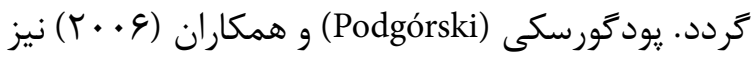

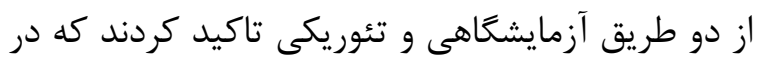

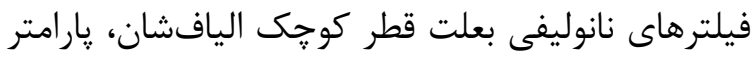

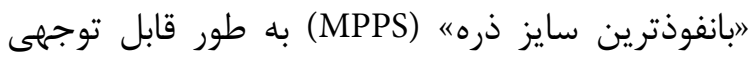

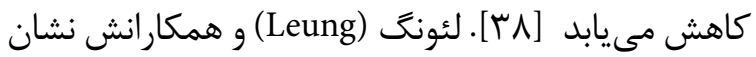

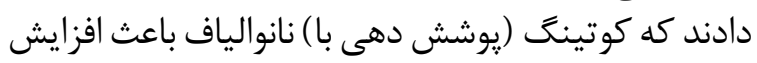

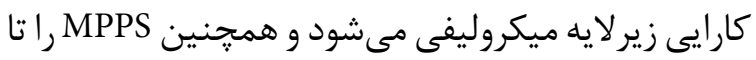

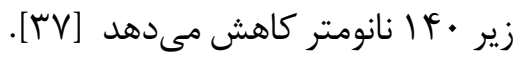

$$
\text { نتيجه كَيرى }
$$

طبق نتايج مطالعه حاضر، با انجام يردازش پيلاسما 
مقايسه عملكرد فيلتراسيون نانوالياف PAN/MgO ساده و پردازش شده با پيلاسما ...

Zadeh AS: Experimental Investigations on electrospun mat production: for use in high-performance air filters. International Journal of Occupational Hygiene 2015, 7(3):110-118.

16. Bettencourt da Silva R, Williams A: Eurachem/CITAC Guide: Setting and Using Target Uncertainty

in Chemical Measurement; 2015.

17. Shao C, Guan H, Liu Y, Mu R: MgO nanofibres via an electrospinning technique. Journal of materials science 2006, 41(12):3821-3824.

18. Dehghan SF, Golbabaei, F.2, Mousavi, T2, Mohammadi, H 2, Kohneshahri, M.H.2 and Bakhtiari, R.3: Production of nanofibers containing magnesium oxide nanoparticles for removing bioaerosol. Pollution 2020, 6(1):185-196.

19. Bao L, Seki K, Niinuma H, Otani Y, Balgis R, Ogi T, Gradon L, Okuyama K: Verification of slip flow in nanofiber filter media through pressure drop measurement at low-pressure conditions. Separation and Purification Technology 2016, 159:100-107.

20. Wang J, Kim SC, Pui DY: Investigation of the figure of merit for filters with a single nanofiber layer on a substrate. Journal of Aerosol Science 2008, 39(4):323-334.

21. Brown RC: Air filtration. London: Pergamon Press; 1993.

22. Hosseini S, Tafreshi HV: Modeling permeability of $3-D$ nanofiber media in slip flow regime. Chemical Engineering Science 2010, 65(6):2249-2254.

23. Karwa AN, Tatarchuk BJ: Aerosol filtration enhancement using carbon nanostructures synthesized within a sintered nickel microfibrous matrix. Separation and purification technology 2012, 87:84-94.

24. Liu J, Zeng B, Wu Z, Zhu J, Liu X: Improved field emission property of graphene paper by plasma treatment. Applied Physics Letters 2010, 97(3):033109.

25. Wei Q: Surface characterization of plasma-treated polypropylene fibers. Materials Characterization 2004, 52(3):231-235.

26. Wang N, Zhu Z, Sheng J, Al-Deyab SS, Yu J, Ding B: Superamphiphobic nanofibrous membranes for effective filtration of fine particles. Journal of colloid and interface science 2014, 428:41-48.

27. Moradi G, Sedighzadeh A, Yarahmadi R, Bakand S, Farshad A, Rezaeifard B: Synthesis of nano-fibrous mats using electrospinning method and determining their efficiency for nanoaerososls removal. Iran Occupational Health 2014, 11(4):1-11.

28. Hutten I: Handbook of Nonwoven Filter Media. Oxford Elsevier; 2007.

29. Papkov D, Zou Y, Andalib MN, Goponenko A, Cheng SZ, Dzenis YA: Simultaneously strong and tough ultrafine continuous nanofibers. ACS nano 2013, 7(4):3324-3331.

30. Zhang Q, Welch J, Park H, Wu C-Y, Sigmund W, trends in nanofibrous membranes and their suitability for air and water filtrations. Membranes 2011, 1(3):232248.

5. Park JH, Yoon KY, Na H, Kim YS, Hwang J, Kim J, Yoon YH: Fabrication of a multi-walled carbon nanotubedeposited glass fiber air filter for the enhancement of nano and submicron aerosol particle filtration and additional antibacterial efficacy. Science of the total environment 2011, 409(19):4132-4138.

6. Dehghan SF, Golbabaei F, Sedigh-Zadeh A, Mohammadi $\mathrm{H}$ : Possibility of using plasma-processed hybrid nanofibers to remove toluene in air stream. Journal of Health and Safety at Work 2019, 9(3):179-190.

7. Vitchuli N, Shi Q, Nowak J, Nawalakhe R, Sieber M, Bourham M, McCord M, Zhang X: Plasmaelectrospinning hybrid process and plasma pretreatment to improve adhesive properties of nanofibers on fabric surface. Plasma Chemistry and Plasma Processing 2012, 32(2):275-291.

8. Kamlangkla K, Paosawatyanyong B, Pavarajarn V, Hodak JH, Hodak SK: Mechanical strength and hydrophobicity of cotton fabric after SF6 plasma treatment. Applied Surface Science 2010, 256(20):5888-5897.

9. Dehghan S, Golbabaei F, Maddah B, Latifi M, Pezeshk H, Hasanzadeh M, Akbar F: Optimization of Electrospinning Parameters for PAN-MgO Nanofibers Applied in Air Filtration Somayeh. Journal of the Air \& Waste Management Association 2016, 66(9):912-921.

10. ISO: ISO 29463: High-efficiency filters and filter media for removing particles in air-- Part 3: Testing flat sheet filter media. In. Geneva: International Organization for Standardization; 2011.

11. ASHREA: ASHREA 52.2: Method of Testing General Ventilation Air-Cleaning Devices for Removal Efficiency by Particle Size. In. Atlanta: American Society of Heating, Refrigerating and Air-Conditioning Engineers, Inc.; 2006.

12. Habibi Mohraz M, Golbabaei F, Je Yu I, Sedigh Zadeh A, Mansournia MA, Farhang Dehghan S: Investigating effective parameters on the nanoparticles air filtration using Polyurethane nanofiber mats. Health and Safety at Work 2018, 8(1):29-42.

13. Matulevicius J, Kliucininkas L, Martuzevicius D, Krugly E, Tichonovas M, Baltrusaitis J: Design and characterization of electrospun polyamide nanofiber media for air filtration applications. Journal of nanomaterials 2014, 2014:14.

14. Dehghan S, Maddah B, Golbabaei F: The Development of Nanofibrous Media Filter Containing Nanoparticles for Removing Particles from Air Stream. Iranian Journal of Health and Environment 2016, 8(4):509-524.

15. Dehghan SF, Golbaaei F, Maddah B, Yarahmadi R, 
35. Kaur S, Gopal R, Ng WJ, Ramakrishna S, Matsuura T: Next-generation fibrous media for water treatment. Mrs Bulletin 2008, 33(1):21-26.

36. Yun KM, Suryamas AB, Iskandar F, Bao L, Niinuma $\mathrm{H}$, Okuyama K: Morphology optimization of polymer nanofiber for applications in aerosol particle filtration. Separation and purification technology 2010, 75(3):340345.

37. Leung WW-F, Hung C-H, Yuen P-T: Effect of face velocity, nanofiber packing density and thickness on filtration performance of filters with nanofibers coated on a substrate. Separation and purification technology 2010, 71(1):30-37.

38. Podgórski A, Bałazy A, Gradoń L: Application of nanofibers to improve the filtration efficiency of the most penetrating aerosol particles in fibrous filters. Chemical Engineering Science 2006, 61(20):6804-6815.
Marijnissen JC: Improvement in nanofiber filtration by multiple thin layers of nanofiber mats. Journal of Aerosol Science 2010, 41(2):230-236.

31. Yu X, Xiang H, Long Y, Zhao N, Zhang X, Xu J: Preparation of porous polyacrylonitrile fibers by electrospinning a ternary system of PAN/DMF/H2O. Materials Letters 2010, 64(22):2407-2409.

32. Nataraj S, Yang K, Aminabhavi T: Polyacrylonitrilebased nanofibers-A state-of-the-art review. Progress in polymer science 2012, 37(3):487-513.

33. Chen H-M, Yu D-G: An elevated temperature electrospinning process for preparing acyclovir-loaded PAN ultrafine fibers. Journal of Materials Processing Technology 2010, 210(12):1551-1555.

34. Ji L, Zhang X: Ultrafine polyacrylonitrile/silica composite fibers via electrospinning. Materials Letters 2008, 62(14):2161-2164. 